Supporting information

\title{
Ligand Effects in Gold- and Platinum-Catalyzed Cyclization of Enynes: Chiral Gold Complexes for the Enantioselective Alkoxycyclization
}

M. Paz Muñoz, Javier Adrio, Juan Carlos Carretero, and Antonio M. Echavarren*

Departamento de Química Orgánica, Universidad Autónoma de Madrid, Cantoblanco, 28049 Madrid, and Institute of Chemical Research of Catalonia (ICIQ), 43007 Tarragona, Spain

\section{General Procedure for Asymmetric Alkoxycyclization of Enynes.}

$\mathbf{P t C l}_{2}$-catalyzed reaction: A mixture of enyne $(0.12 \mathrm{mmol})$, the chiral ligand $(5-10 \mathrm{~mol} \%)$, and $\mathrm{PtCl}_{2}(5 \mathrm{~mol} \%)$ in $\mathrm{MeOH}(5 \mathrm{~mL})$ was heated under reflux for the stated time. The solvent was evaporated, and the crude mixture was purified by flash chromatography (hexane-EtOAc mixtures) to give the corresponding carbocycles.

[Au( $\left.\left(\mathbf{L}^{*}\right) \mathbf{C l}\right]$-catalyzed reaction: A mixture of $\left[\mathrm{Au}\left(\mathrm{L}^{*}\right) \mathrm{Cl}\right](2 \mathrm{~mol} \%)$ or $\left[\mathrm{L}^{*}(\mathrm{AuCl})_{2}\right](1.6$ mol\%), and $\mathrm{AgSbF}_{6}(2 \mathrm{~mol} \%)$ in $\mathrm{MeOH}(1 \mathrm{~mL})$ was stirred at room temperature for $5 \mathrm{~min}$. The enyne $(0.12 \mathrm{mmol})$ was added in $\mathrm{MeOH}(2 \mathrm{~mL})$ and the reaction mixture was stirred at room temperature for the stated time. The mixture was filtered through $\mathrm{SiO}_{2}$, and the solvent was evaporated. The crude mixture was purified by flash chromatography (hexane-EtOAc mixtures) to give the carbocycles.

The enantiomeric excesses were determined at $25^{\circ} \mathrm{C}$ in Perkin-Elmer Integral 4000 or Agilent 1100 Series HPLC apparatus. The chiral column and conditions were detailed in each case.

Racemic 8, 9, 17, 18, 32, 51, 53, 55 and 56 have been described before. ${ }^{1}$

Dimethyl-3-(1-methoxy-1-methylethyl)-4-methylenecyclopentane 1,1-dicarboxylate (8). Chiral Column: Daicel ChiralPack AD; Conditions: 95:5 hexane- $i$-PrOH, flow $=0.5 \mathrm{~mL} / \mathrm{min}$; $\lambda=220 \mathrm{~nm}$. Retention times: $9.8 \mathrm{~min}$, and $11.4 \mathrm{~min}$.

\section{1,1-Bis(phenylsulfonyl)-3-(1-methoxy-1-methylethyl)-4-methylenecyclopentane}

Chiral Column: Daicel ChiralPack AD; Conditions: 90:10 hexane-i-PrOH, flow $=0.7$ $\mathrm{mL} / \mathrm{min} ; \lambda=220 \mathrm{~nm}$. Retention times: $29.8 \mathrm{~min}$, and $35.4 \mathrm{~min} .53 \%$ ee: $[\alpha]^{25}=+21.2(\mathrm{c}=0.4$, $\left.\mathrm{CHCl}_{3}\right)$. 


\section{1,1-Bis(phenylsulfonyl)-(3R*)-[(1S*)-1-(methoxy)phenylmethyl]-4-}

methylenecyclopentane (17). Chiral Column: Daicel ChiralPack AD; Conditions: 90:10 hexane- $i-\mathrm{PrOH}$, flow $=0.7 \mathrm{~mL} / \mathrm{min} ; \lambda=220 \mathrm{~nm}$. Retention times: $35.2 \mathrm{~min}$, and $38.4 \mathrm{~min}$. $49 \%$ ee: $[\alpha]^{25}=+21.3\left(\mathrm{c}=0.3, \mathrm{CHCl}_{3}\right)$.

\section{1,1-Bis(phenylsulfonyl)-(3R*)-[(1S*)-1-(allyloxy)phenylmethyl]-4-}

methylenecyclopentane (51). Chiral Column: Daicel ChiralPack AD; Conditions: 90:10 hexane- $i$-PrOH, flow $=0.7 \mathrm{~mL} / \mathrm{min} ; \lambda=220 \mathrm{~nm}$. Retention times: $27.9 \mathrm{~min}$, and $29.7 \mathrm{~min}$. $36 \%$ ee: $[\alpha]^{25}=+8.5\left(\mathrm{c}=0.2, \mathrm{CHCl}_{3}\right)$.

\section{(Z)-1,1-Bis(phenylsulfonyl)-3-benzylidene-4-(1-methoxy-1-methylethyl)cyclopentane}

(53). Chiral Column: Daicel ChiralPack AS; Conditions: 90:10 hexane- $i$-PrOH, flow $=1$ $\mathrm{mL} / \mathrm{min} ; \lambda=220 \mathrm{~nm}$. Retention times: $14.4 \mathrm{~min}$, and $18.9 \mathrm{~min} .94 \%$ ee: $[\alpha]^{25}=-75.3(\mathrm{c}=0.6$, $\left.\mathrm{CHCl}_{3}\right)$.

Dimethyl 3-methyl-3-methoxy-5-methylenecyclohexane-1,1-dicarboxylate (55). Chiral Column: Daicel ChiralPack AD; Conditions: 99:1 hexane- $i-\mathrm{PrOH}$, flow $=0.7 \mathrm{~mL} / \mathrm{min} ; \lambda=$ $220 \mathrm{~nm}$. Retention times: $13.8 \mathrm{~min}$, and $15.2 \mathrm{~min} .38 \%$ ee: $[\alpha]^{25}=+7.7\left(\mathrm{c}=0.6, \mathrm{CHCl}_{3}\right)$.

\section{Pd-catalyzed deallylation of 51 (Eq 1)}

To a solution of $51(95 \mathrm{mg}, 0.19 \mathrm{mmol})$ in HOAc $(1.5 \mathrm{~mL})$ and water (2 drops) at room temperature were added $\mathrm{PdCl}_{2}(85 \mathrm{mg}, 0.48 \mathrm{mmol})$ and $\mathrm{NaOAc}(86 \mathrm{mg}, 1.05 \mathrm{mmol})$. The mixture was then heated at $50^{\circ} \mathrm{C}$ for $20 \mathrm{~h}$. After being cooled to room temperature, the mixture was filtered through Celtite and washed with $\mathrm{Et}_{2} \mathrm{O}$. The solution was then washed with $\mathrm{NaHCO}_{3}$, and evaporated. After flash chromatography (5:1 hexane-EtOAc,), alcohol 56 was obtained as a white solid (49 $\mathrm{mg}, 57 \%)$.

\section{Formation of Mosher eters of 56}

A solution of 56 (49 mg, $0.10 \mathrm{mmol})$ and (R)-(-)-MTPACl (40 $\mathrm{mg}, 0.15 \mathrm{mmol})$ was stirred in piridine $(0.7 \mathrm{~mL})$ for $2 \mathrm{~h}$. After extractive work-up $\left(\mathrm{Et}_{2} \mathrm{O}-10 \%\right.$ aqueous $\left.\mathrm{HCl}\right)$ and chromatography (10:1 hexane-EtOAc,), the Mosher esters were obtained as a 2.1:1 mixture (41 mg, 60\%).

\section{Determination of the Configuration of 56}

Selected ${ }^{1} \mathrm{H}$ - and ${ }^{13} \mathrm{C}-\mathrm{NMR}$ shifts for the Mosher esters of $\mathbf{5 6}$ are given below: 


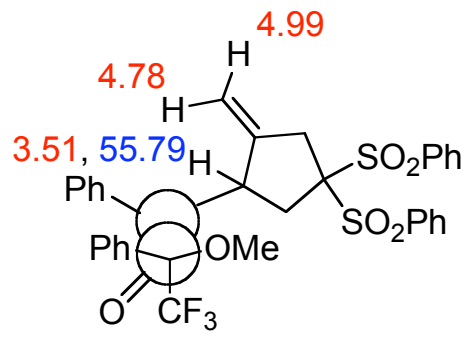

major isomer

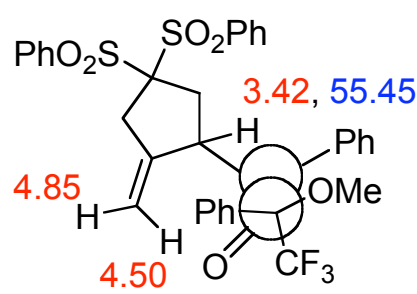

minor isomer

Analysis of the more stable conformations (MM2 + PM3 calculations) in which $\mathrm{C}\left(1^{\prime}\right) \mathrm{H}$, the carbonyl group, and the $\mathrm{CF}_{3}$ substituent are in the approximately in the plane, indicates that the minor isomer should show the $\mathrm{H}$ at $\mathrm{C}-4$ shielded by the phenyl group pf the MTPA.

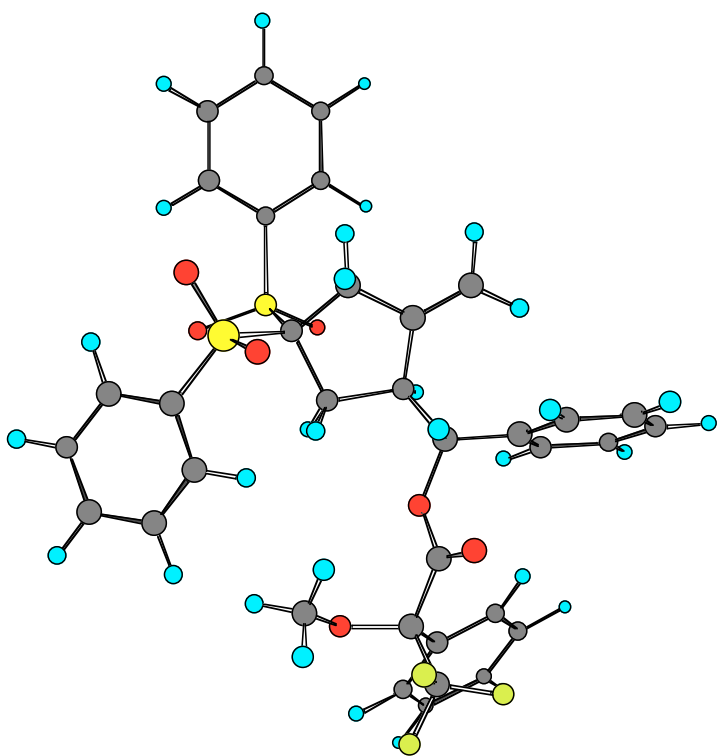

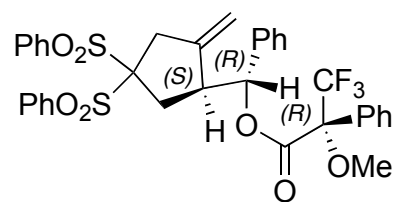

major isomer

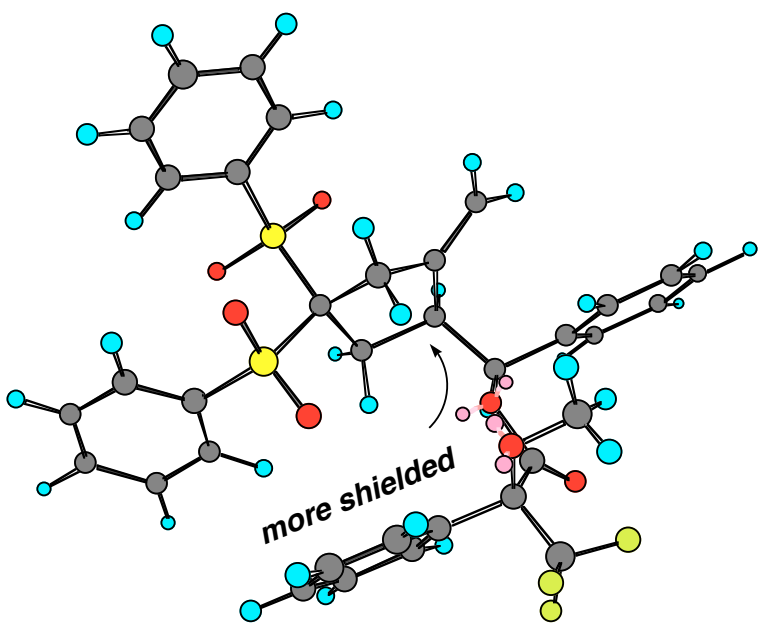

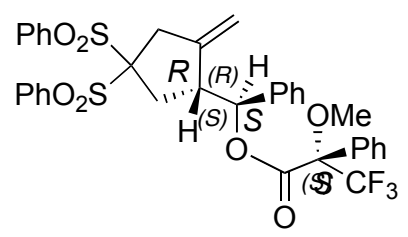

minor isomer 


\section{Synthesis of Chiral Metal Complexes}

\section{Dichloro-[(S)-(-)-4-isopropyl-2-(2-pyridinyl)-2-oxazoline]platinum(II) (30)}

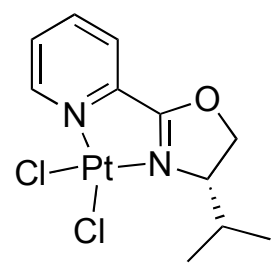

A suspension of (S)-(-)-4-isopropyl-2-(2-pyridinyl)-2-oxazoline ${ }^{2}(71 \mathrm{mg}, 0.38 \mathrm{mmol})$ and $\mathrm{PtCl}_{2}(100 \mathrm{mg}, 0.38 \mathrm{mmol})$ was stirred in $\mathrm{MeOH}(10 \mathrm{~mL})$ under reflux for $24 \mathrm{~h}$. The reaction mixture was cooled to room temperature and the solvent was evaporated. The crude was triturated with $\mathrm{Et}_{2} \mathrm{O}$ at $-20^{\circ} \mathrm{C}$ to give complex 30 as an orange solid $(85 \mathrm{mg}, 49 \%):{ }^{1} \mathrm{H}$ NMR $\left(\mathrm{CDCl}_{3}, 300 \mathrm{MHz}\right) \delta 9.26(\mathrm{~d}, J=5.45 \mathrm{~Hz}, 1 \mathrm{H}), 8.15(\mathrm{t}, J=7.7 \mathrm{~Hz}, 1 \mathrm{H}), 7.75(\mathrm{~d}, J=7.7 \mathrm{~Hz}$, 1H), $7.57(\mathrm{~m}, 1 \mathrm{H}), 5.12(\mathrm{t}, J=9.3 \mathrm{~Hz}, 1 \mathrm{H}), 4.82(\mathrm{dd}, J=8.7,4.8 \mathrm{~Hz}, 1 \mathrm{H}), 4.74(\mathrm{~m}, 1 \mathrm{H}), 2.95$ $(\mathrm{qd}, J=6.9,3.0 \mathrm{~Hz}, 1 \mathrm{H}), 0.96(\mathrm{~d}, J=7.1 \mathrm{~Hz}, 3 \mathrm{H}), 0.80(\mathrm{~d}, J=6.9 \mathrm{~Hz}, 3 \mathrm{H}) ;{ }^{13} \mathrm{C} \mathrm{NMR}(75$ $\mathrm{MHz}, \mathrm{CDCl}_{3}$; DEPT) $\delta 172.90(\mathrm{C}), 149.01(\mathrm{CH}), 144.80(\mathrm{C}), 139.14(\mathrm{CH}), 129.18(\mathrm{CH})$, $126.29(\mathrm{CH}), 73.03\left(\mathrm{CH}_{2}\right), 67.96(\mathrm{CH}), 28.71(\mathrm{CH}), 18.65\left(\mathrm{CH}_{3}\right), 13.81\left(\mathrm{CH}_{3}\right)$; Anal. Calcd for $\mathrm{C}_{11} \mathrm{H}_{14} \mathrm{Cl}_{2} \mathrm{~N}_{2} \mathrm{OPt} \cdot \mathrm{H}_{2} \mathrm{O}$ : C, 27.86; H, 3.40; N, 5.91. Found: C, 28.02; H, 3.45; N, 6.01. HRMS-EI Calcd for $\mathrm{C}_{11} \mathrm{H}_{14} \mathrm{Cl}_{2} \mathrm{~N}_{2} \mathrm{OPt}$ : $456.0209\left(\mathrm{M}^{+}+1\right)$. Found: 456.0217.

General procedure for the synthesis of chiral $\mathbf{A u}(\mathbf{I})$ complexes: To a solution of $\mathrm{Na}\left[\mathrm{AuCl}_{4}\right] \cdot \mathrm{H}_{2} \mathrm{O}(0.05-0.22 \mathrm{mmol})$ in $\mathrm{H}_{2} \mathrm{O}(2-7 \mathrm{~mL})$ at $0^{\circ} \mathrm{C}$, was added 2,2'-thiodiethanol (0.05-0.22 mmol) over $15 \mathrm{~min}$. After stirring for $1 \mathrm{~h}$, the ligand (0.15-0.44 mmol) was added in $\mathrm{CHCl}_{3}(0.5-2 \mathrm{~mL})$ at $0^{\circ} \mathrm{C}$, and the resulting mixture was stirred for a $1 \mathrm{~h}$. After extractive work-up $\left(\mathrm{CHCl}_{3}\right)$ and trituration (hexane) corresponding $\mathrm{Au}(\mathrm{I})$ complexes are obtained.

Chloro-(R)-2-(tert-Butylsulfenyl)-1-(diphenylphosphino)ferrocene gold(I) (39)

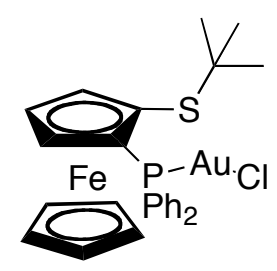

Yellow solid (96\%): $\mathrm{mp}>190{ }^{\circ} \mathrm{C}(\mathrm{dec}) ;[\alpha]^{25}=-103\left(\mathrm{c}=1, \mathrm{CHCl}_{3}\right) ;{ }^{1} \mathrm{H}$ NMR $(300 \mathrm{MHz}$, $\left.\mathrm{CDCl}_{3}\right) \delta 7.86\left(\mathrm{ddd},{ }^{3} J\left({ }^{1} \mathrm{H}_{-}{ }^{31} \mathrm{P}\right)=13.3, J\left({ }^{1} \mathrm{H}_{-}{ }^{1} \mathrm{H}\right)=7.7,1.2 \mathrm{~Hz}, 2 \mathrm{H}\right), 7.56\left(\mathrm{ddd},{ }^{3} J\left({ }^{1} \mathrm{H}_{-}{ }^{31} \mathrm{P}\right)=\right.$ $\left.13.1, J\left({ }^{1} \mathrm{H}^{-1} \mathrm{H}\right)=7.7,1.2 \mathrm{~Hz}, 2 \mathrm{H}\right), 7.50(\mathrm{t}, J=7.5 \mathrm{~Hz}, 3 \mathrm{H}), 7.39-7.33(\mathrm{~m}, 3 \mathrm{H}), 4.84$ (quint, $J=$ 
$1.0 \mathrm{~Hz}, 1 \mathrm{H}), 4.63(\mathrm{t}, J=2.5 \mathrm{~Hz}, 1 \mathrm{H}), 4.28(\mathrm{q}, J=1.2 \mathrm{~Hz}, 1 \mathrm{H}), 4.07(\mathrm{~s}, 5 \mathrm{H}), 1.01(\mathrm{~s}, 9 \mathrm{H}) ;{ }^{13} \mathrm{C}$ $\operatorname{NMR}\left(75 \mathrm{MHz}, \mathrm{CDCl}_{3}\right) \delta 134.78\left(\mathrm{~d}, J\left({ }^{13} \mathrm{C}-{ }^{31} \mathrm{P}\right)=14.6 \mathrm{~Hz}, \mathrm{PPh}_{2}\right), 133.11\left(\mathrm{~d}, J\left({ }^{13} \mathrm{C}-{ }^{31} \mathrm{P}\right)=14.6\right.$ $\left.\mathrm{Hz}, \mathrm{PPh}_{2}\right), 132.40,131.71\left(\mathrm{~d}, J\left({ }^{13} \mathrm{C}_{-}{ }^{31} \mathrm{P}\right)=2.1 \mathrm{~Hz}, \mathrm{PPh}_{2}\right), 131.58\left(\mathrm{~d}, J\left({ }^{13} \mathrm{C}_{-}{ }^{31} \mathrm{P}\right)=10.5 \mathrm{~Hz}\right.$, $\left.\mathrm{PPh}_{2}\right), 131.08\left(\mathrm{~d}, J\left({ }^{13} \mathrm{C}-{ }^{31} \mathrm{P}\right)=2.1 \mathrm{~Hz}, \mathrm{PPh}_{2}\right), 130.59,128.71\left(\mathrm{~d}, J\left({ }^{13} \mathrm{C}-{ }^{31} \mathrm{P}\right)=11.5 \mathrm{~Hz}, \mathrm{PPh}_{2}\right)$, $128.65\left(\mathrm{~d}, J\left({ }^{13} \mathrm{C}_{-}{ }^{31} \mathrm{P}\right)=12.5 \mathrm{~Hz}, \mathrm{PPh}_{2}\right), 81.96\left(\mathrm{~d}, J\left({ }^{13} \mathrm{C}^{-31} \mathrm{P}\right)=6.3 \mathrm{~Hz}\right), 74.10\left(\mathrm{~d}, J\left({ }^{13} \mathrm{C}_{-}^{31} \mathrm{P}\right)=\right.$ $5.2 \mathrm{~Hz}$ ), 71.71, 71.69, 47.46, 30.98; ${ }^{31} \mathrm{P}$ NMR (300 MHz, $\left.\mathrm{CDCl}_{3}\right) \delta 22.40$ (s). HRMS-TOFES-MS Calcd for $\mathrm{C}_{26} \mathrm{H}_{27} \mathrm{AuClFePS}$ : $690.0275\left(\mathrm{M}^{+}\right)$. Found: 690.0241. Anal. Calcd for $\mathrm{C}_{26} \mathrm{H}_{27} \mathrm{AuClFePS} \cdot \mathrm{H}_{2} \mathrm{O}: \mathrm{C}, 44.06 ; \mathrm{H}, 4$.12. Found: $\mathrm{C}, 44.46 ; \mathrm{H}, 3.90$.

Chloro-(R)-2-(tert-Butylsulfenyl)-1-(dicyclohexylphosphino)ferrocene gold(I) (40)

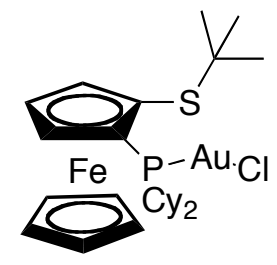

Orange solid: $\mathrm{mp} 82-83{ }^{\circ} \mathrm{C} ;[\alpha]^{25}=+75.8\left(\mathrm{c}=0.53, \mathrm{CHCl}_{3}\right) ;{ }^{1} \mathrm{H} \mathrm{NMR}\left(300 \mathrm{MHz}, \mathrm{CDCl}_{3}\right) \delta$ $4.84(\mathrm{q}, J=1.2 \mathrm{~Hz}, 1 \mathrm{H}), 4.57(\mathrm{td}, J=2.6,1.0 \mathrm{~Hz}, 1 \mathrm{H}), 4.51(\mathrm{td}, J=3.0,1.5 \mathrm{~Hz}, 1 \mathrm{H}), 4.32(\mathrm{~s}$, 5H), $2.6(\mathrm{~m}, 1 \mathrm{H}), 2.30(\mathrm{~m}, 1 \mathrm{H}), 2.10(\mathrm{~m}, 4 \mathrm{H}), 1.85(\mathrm{~m}, 4 \mathrm{H}), 1.72(\mathrm{~m}, 1 \mathrm{H}), 1.57-1.55(\mathrm{~m}, 6 \mathrm{H})$, 1.35-1.29 (m, 5H), $1.30(\mathrm{~s}, 9 \mathrm{H}) ;{ }^{13} \mathrm{C}$ NMR (75 MHz, CDCl 3 ; DEPT) $\delta 81.97(\mathrm{C}), 79.47(\mathrm{~d}$, $\left.J\left({ }^{13} \mathrm{C}-{ }^{31} \mathrm{P}\right)=5.6 \mathrm{~Hz}, \mathrm{CH}\right), 72.66(\mathrm{C}), 71.84(\mathrm{CH}), 47.17(\mathrm{C}), 37.00\left(\mathrm{~d}, J\left({ }^{13} \mathrm{C}-{ }^{31} \mathrm{P}\right)=33.5 \mathrm{~Hz}\right)$, $36.10\left(\mathrm{~d}, J\left({ }^{13} \mathrm{C}_{-}{ }^{31} \mathrm{P}\right)=35.0 \mathrm{~Hz}\right), 33.61\left(\mathrm{~m}, \mathrm{CH}_{2}\right), 31.88(\mathrm{CH}), 31.31\left(\mathrm{CH}_{3}\right), 31.20(\mathrm{CH}), 29.26$ $\left(\mathrm{CH}_{2}\right), 27.20\left(\mathrm{CH}_{2}\right), 27.00\left(\mathrm{CH}_{2}\right), 26.91\left(\mathrm{CH}_{2}\right), 26.74\left(\mathrm{CH}_{2}\right), 26.67\left(\mathrm{CH}_{2}\right), 26.57\left(\mathrm{CH}_{2}\right), 25.64$ $\left(\mathrm{CH}_{2}\right) ;{ }^{31} \mathrm{P}$ NMR (121.5 MHz, $\left.\mathrm{CDCl}_{3}\right) \quad \delta \quad 44.85$ (s). HRMS-TOF-ES-MS Calcd for $\mathrm{C}_{26} \mathrm{H}_{39} \mathrm{AuClFePS}$ : $702.1214\left(\mathrm{M}^{+}\right)$. Found: 702.1186.

\section{Chloro-( $\left.R_{\mathrm{Fc}}, R_{\mathrm{S}}\right)$-1-(tert-Butylsulfinyl)-2-(diphenylphosphino)-ferrocene gold(I) (41)}

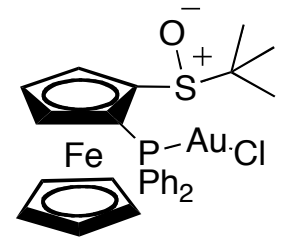

Orange solid: $\mathrm{mp}>190{ }^{\circ} \mathrm{C}(\mathrm{dec}) ;[\alpha]^{25}=-83.2\left(\mathrm{c}=0.40, \mathrm{CHCl}_{3}\right) ;{ }^{1} \mathrm{H}$ NMR $(300 \mathrm{MHz}$, 
$\left.\mathrm{CDCl}_{3}\right) \delta 7.80\left(\mathrm{ddd},{ }^{3} J\left({ }^{1} \mathrm{H}_{-}-{ }^{31} \mathrm{P}\right)=13.3, J\left({ }^{1} \mathrm{H}_{-}{ }^{1} \mathrm{H}\right)=5.3,1.6 \mathrm{~Hz}, 2 \mathrm{H}\right), 7.60\left(\mathrm{ddd},{ }^{3} J\left({ }^{1} \mathrm{H}_{-}{ }^{31} \mathrm{P}\right)=\right.$ 13.3, $\left.J\left({ }^{1} \mathrm{H}-{ }^{1} \mathrm{H}\right)=7.7,1.2 \mathrm{~Hz}, 2 \mathrm{H}\right), 7.54-7.35(\mathrm{~m}, 6 \mathrm{H}), 4.78(\mathrm{q}, J=1.6 \mathrm{~Hz}, 1 \mathrm{H}), 4.64(\mathrm{t}, J=2.6$ $\mathrm{Hz}, 1 \mathrm{H}), 4.46$ (s, 5H), $4.03(\mathrm{td}, J=2.8,1.6 \mathrm{~Hz}, 1 \mathrm{H}), 1.08(\mathrm{~s}, 9 \mathrm{H}) ;{ }^{13} \mathrm{C} \mathrm{NMR}\left(75 \mathrm{MHz}, \mathrm{CDCl}_{3}\right)$ $\delta 134.95\left(\mathrm{~d}, J\left({ }^{13} \mathrm{C}_{-}{ }^{31} \mathrm{P}\right)=15.7 \mathrm{~Hz}, \mathrm{PPh}_{2}\right), 133.79\left(\mathrm{~d}, J\left({ }^{13} \mathrm{C}_{-}{ }^{31} \mathrm{P}\right)=14.6 \mathrm{~Hz}, \mathrm{PPh}_{2}\right), 131.62(\mathrm{~d}$, $\left.J\left({ }^{13} \mathrm{C}-{ }^{31} \mathrm{P}\right)=12.5 \mathrm{~Hz}, \mathrm{PPh}_{2}\right), 130.69\left(\mathrm{~d}, J\left({ }^{13} \mathrm{C}-{ }^{31} \mathrm{P}\right)=20.9 \mathrm{~Hz}, \mathrm{PPh}_{2}\right), 128.76,128.62\left(\mathrm{~d}, J\left({ }^{13} \mathrm{C}-\right.\right.$ $\left.\left.{ }^{31} \mathrm{P}\right)=8.1 \mathrm{~Hz}, \mathrm{PPh}_{2}\right), 75.96\left(\mathrm{~d}, J\left({ }^{13} \mathrm{C}-{ }^{31} \mathrm{P}\right)=4.2 \mathrm{~Hz}\right), 73.67\left(\mathrm{~d}, J\left({ }^{13} \mathrm{C}-{ }^{31} \mathrm{P}\right)=6.3 \mathrm{~Hz}\right), 72.80(\mathrm{~d}$, $\left.J\left({ }^{13} \mathrm{C}-{ }^{31} \mathrm{P}\right)=6.3 \mathrm{~Hz}\right), 72.65,71.78,57.23,23.84 ;{ }^{31} \mathrm{P} \mathrm{NMR}\left(300 \mathrm{MHz}, \mathrm{CDCl}_{3}\right) \delta 27.43(\mathrm{~s})$. HRMS-TOF-ES-MS Calcd for $\mathrm{C}_{26} \mathrm{H}_{27} \mathrm{AuClFePS} \cdot \mathrm{Na}$ : $729.0121\left(\mathrm{M}^{+}\right)$. Found: 729.0092.

\section{Gold(I)-complex 42}

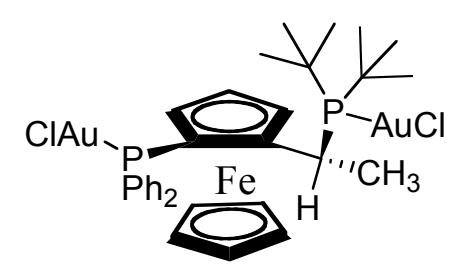

Yellow solid: $\mathrm{mp}>200{ }^{\circ} \mathrm{C}(\mathrm{dec}) ;[\alpha]^{25}=-36.7\left(\mathrm{c}=0.63, \mathrm{CHCl}_{3}\right) ;{ }^{1} \mathrm{H}$ NMR $(300 \mathrm{MHz}$, $\left.\mathrm{CDCl}_{3}\right) \delta 7.81\left(\mathrm{dd},{ }^{3} J\left({ }^{1} \mathrm{H}_{-}{ }^{31} \mathrm{P}\right)=12.5, J\left({ }^{1} \mathrm{H}-{ }^{1} \mathrm{H}\right)=7.3 \mathrm{~Hz}, 2 \mathrm{H}\right), 7.70\left(\mathrm{dd},{ }^{3} J\left({ }^{1} \mathrm{H}^{3}{ }^{31} \mathrm{P}\right)=12.5\right.$, $\left.J\left({ }^{1} \mathrm{H}^{-1} \mathrm{H}\right)=7.3 \mathrm{~Hz}, 2 \mathrm{H}\right), 7.53-7.40(\mathrm{~m}, 6 \mathrm{H}), 4.80($ br s, $1 \mathrm{H}), 4.66($ br s, $1 \mathrm{H}), 4.17($ br s, $1 \mathrm{H})$, $4.10(\mathrm{~s}, 5 \mathrm{H}), 2.13\left(\mathrm{dd},{ }^{3} J\left({ }^{1} \mathrm{H}^{31}{ }^{31} \mathrm{P}\right)=10.7, J\left({ }^{1} \mathrm{H}-{ }^{1} \mathrm{H}\right)=7.7 \mathrm{~Hz}, 1 \mathrm{H}\right), 1.55\left(\mathrm{~d},{ }^{3} J\left({ }^{1} \mathrm{H}^{3}{ }^{31} \mathrm{P}\right)=14.8\right.$ $\mathrm{Hz}, 9 \mathrm{H}+3 \mathrm{H}), 1.01\left(\mathrm{~d},{ }^{3} J\left({ }^{1} \mathrm{H}_{-}{ }^{31} \mathrm{P}\right)=14.8 \mathrm{~Hz}, 9 \mathrm{H}\right) ;{ }^{13} \mathrm{C} \mathrm{NMR}\left(75 \mathrm{MHz}, \mathrm{CDCl}_{3}\right) \delta 135.32(\mathrm{~d}$, $\left.{ }^{2} J\left({ }^{13} \mathrm{C}-{ }^{31} \mathrm{P}\right)=15.7 \mathrm{~Hz}, \mathrm{PPh}_{2}\right), 133.60\left(\mathrm{~d},{ }^{2} J\left({ }^{13} \mathrm{C}-{ }^{31} \mathrm{P}\right)=14.6 \mathrm{~Hz}, \mathrm{PPh}_{2}\right), 131.91,131.30,129.70$ $\left(\mathrm{d},{ }^{3} J\left({ }^{13} \mathrm{C}-{ }^{31} \mathrm{P}\right)=11.5 \mathrm{~Hz}, \mathrm{PPh}_{2}\right), 128.85\left(\mathrm{~d},{ }^{3} J\left({ }^{13} \mathrm{C}-{ }^{31} \mathrm{P}\right)=11.5 \mathrm{~Hz}, \mathrm{PPh}_{2}\right), 77.19,76.09,75.66$, $72.18\left(\mathrm{~d}, J\left({ }^{13} \mathrm{C}-{ }^{31} \mathrm{P}\right)=5.2 \mathrm{~Hz}\right), 71.70\left(\mathrm{~d}, J\left({ }^{13} \mathrm{C}-{ }^{31} \mathrm{P}\right)=7.3 \mathrm{~Hz}\right), 70.68,38.45\left(\mathrm{~d}, J\left({ }^{13} \mathrm{C}-{ }^{31} \mathrm{P}\right)=\right.$ $17.8 \mathrm{~Hz}), 37.70\left(\mathrm{~d}, J\left({ }^{13} \mathrm{C}-{ }^{31} \mathrm{P}\right)=23.0 \mathrm{~Hz}\right), 31.89\left(\mathrm{~d}, J\left({ }^{13} \mathrm{C}-{ }^{31} \mathrm{P}\right)=5.2 \mathrm{~Hz}\right), 30.97(\mathrm{br} \mathrm{s}), 29.44$ $\left(\mathrm{d}, J\left({ }^{13} \mathrm{C}-{ }^{31} \mathrm{P}\right)=5.2 \mathrm{~Hz}\right), 23.94\left(\right.$ br s) $;{ }^{31} \mathrm{P} \mathrm{NMR}\left(121.5 \mathrm{MHz}, \mathrm{CDCl}_{3}\right) \delta 87.47$ (s), 87.35 (s). Anal. Calcd for $\mathrm{C}_{32} \mathrm{H}_{40} \mathrm{Au}_{2} \mathrm{Cl}_{2} \mathrm{Fe}_{2} \mathrm{P}_{2}$ : C, 38.16; H, 4.00. Found: C, 38.65; H, 4.08.

\section{Gold(I)-complex 43}

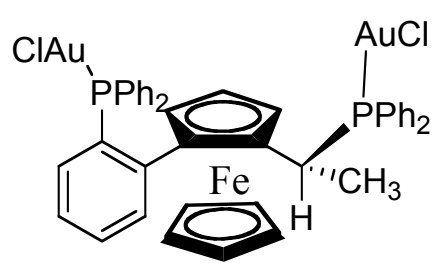


Orange solid: $\mathrm{mp}>200{ }^{\circ} \mathrm{C} ;[\alpha]^{25}=-107.3\left(\mathrm{c}=0.30, \mathrm{CHCl}_{3}\right) ;{ }^{1} \mathrm{H}$ NMR $\left(300 \mathrm{MHz}, \mathrm{CDCl}_{3}\right) \delta$ $8.35(\mathrm{dd}, J=6.9,5.1 \mathrm{~Hz}, 1 \mathrm{H}), 7.74(\mathrm{t}, J=7.6 \mathrm{~Hz}, 1 \mathrm{H}), 7.57-7.29(\mathrm{~m}, 21 \mathrm{H}), 6.68(\mathrm{dd}, J=12.5$ , $7.6 \mathrm{~Hz}, 1 \mathrm{H}), 4.23(\mathrm{t}, J=2.6 \mathrm{~Hz}, 1 \mathrm{H}), 4.05(\mathrm{~m}, 1 \mathrm{H}), 3.99(\mathrm{~s}, 1 \mathrm{H}), 3.98(\mathrm{~s}, 5 \mathrm{H}), 3.95(\mathrm{~s}, 1 \mathrm{H})$, $1.7(\mathrm{dd}, J=17.4,7.1 \mathrm{~Hz}, 3) ;{ }^{13} \mathrm{C} \mathrm{NMR}\left(75 \mathrm{MHz}, \mathrm{CDCl}_{3}\right) \delta 135.84\left(\mathrm{~d}, J\left({ }^{13} \mathrm{C}-{ }^{31} \mathrm{P}\right)=14.6 \mathrm{~Hz}\right)$, $135.18,135.05,134.93,134.88,134.70,134.46\left(\mathrm{~d}, J\left({ }^{13} \mathrm{C}-{ }^{31} \mathrm{P}\right)=12.5 \mathrm{~Hz}\right), 133.51\left(\mathrm{~d}, J\left({ }^{13} \mathrm{C}-\right.\right.$ $\left.\left.{ }^{31} \mathrm{P}\right)=13.6 \mathrm{~Hz}\right), 131.74,131.63,130.83,129.68,129.35\left(\mathrm{~d}, J\left({ }^{13} \mathrm{C}-{ }^{31} \mathrm{P}\right)=11.5 \mathrm{~Hz}\right), 129.01$, $128.87,128.72,126.5\left(\mathrm{~d}, J\left({ }^{13} \mathrm{C}-{ }^{31} \mathrm{P}\right)=10.0 \mathrm{~Hz}\right), 70.57,70.14,70.08,69.98,67.78 ;{ }^{31} \mathrm{P}$ NMR $\left(121.5 \mathrm{MHz}, \quad \mathrm{CDCl}_{3}\right) \quad \delta \quad 45.15$ (s), $26.05 \quad$ (s). HRMS-TOF-ES-MS Calcd for $\mathrm{C}_{42} \mathrm{H}_{36} \mathrm{Au}_{2} \mathrm{Cl}_{2} \mathrm{FeP}_{2}$ : $1122.0350 \quad\left(\mathrm{M}^{+}\right)$. Found: 1122.03090. Anal Calcd for $\mathrm{C}_{42} \mathrm{H}_{36} \mathrm{Au}_{2} \mathrm{Cl}_{2} \mathrm{FeP}_{2} \cdot 2 \mathrm{H}_{2} \mathrm{O}$ : C, 43.51; H, 3.48. Found: C, 43.64; H, 3.31 .

\section{Gold(I)-complexes 44}

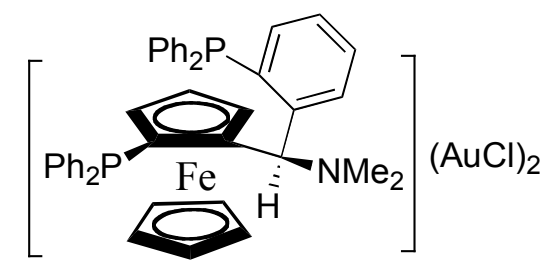

Orange solid: $\mathrm{mp}=167-168^{\circ} \mathrm{C} ;[\alpha]^{25}=+128.4\left(\mathrm{c}=0.50, \mathrm{CHCl}_{3}\right) ;{ }^{31} \mathrm{P} \mathrm{NMR}(300 \mathrm{MHz}$, $\left.\mathrm{CDCl}_{3}\right) \delta 31.35(\mathrm{~d}, J=310.8 \mathrm{~Hz}$, part A AB system), $27.30(\mathrm{~d}, J=313.9 \mathrm{~Hz}$, part B AB system), 24.90 (br s), 23.25 (s). MALDI-TOF-MS: m/z: $1115.9\left(\mathrm{M}^{+}-\mathrm{Cl}\right), 884.1\left(\mathrm{M}^{+}-\mathrm{AuCl}_{2}\right)$.

Chloro $\left[O, O^{\prime}-(\mathrm{R})-\left(1,1^{\prime}\right.\right.$-dinaphtyl-2,2'-diyl)- $N, N$-di-i-propyl-phosphoramidite $]$ gold(I) (45)

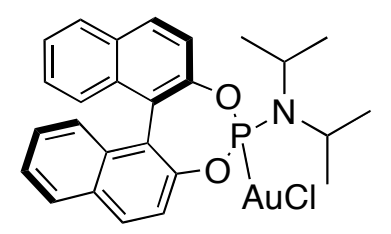

White solid: $\mathrm{mp}>200^{\circ} \mathrm{C} ;[\alpha]^{25}=-258.8\left(\mathrm{c}=0.50, \mathrm{CHCl}_{3}\right) ;{ }^{1} \mathrm{H}$ NMR $\left(300 \mathrm{MHz}, \mathrm{CDCl}_{3}\right) \delta$ $8.07(\mathrm{~d}, J=8.5 \mathrm{~Hz}, 1 \mathrm{H}), 8.00-7.94(\mathrm{~m}, 3 \mathrm{H}), 7.26(\mathrm{~d}, J=8.9 \mathrm{~Hz}, 1 \mathrm{H}), 7.52-7.22(\mathrm{~m}, 7 \mathrm{H}), 3.63$ $(\mathrm{dq}, J=17.0,7.3 \mathrm{~Hz}, 2 \mathrm{H}), 1.40(\mathrm{~d}, J=6.9 \mathrm{~Hz}, 3 \mathrm{H}), 1.29(\mathrm{~d}, J=6.4 \mathrm{~Hz}, 3 \mathrm{H}) ;{ }^{13} \mathrm{C}$ NMR $(75$ $\left.\mathrm{MHz}, \mathrm{CDCl}_{3}\right) \delta 147.84,147.23\left(\mathrm{~d}, J\left({ }^{13} \mathrm{C}-{ }^{31} \mathrm{P}\right)=6.3 \mathrm{~Hz}\right), 132.42-131.24\left(\mathrm{~d}, J\left({ }^{13} \mathrm{C}-{ }^{31} \mathrm{P}\right)=87.8\right.$ $\mathrm{Hz}), 132.05,131.80-130.52\left(\mathrm{~d}, J\left({ }^{13} \mathrm{C}^{-31} \mathrm{P}\right)=96.2 \mathrm{~Hz}\right), 128.53\left(\mathrm{~d}, J\left({ }^{13} \mathrm{C}_{-}{ }^{31} \mathrm{P}\right)=23.0 \mathrm{~Hz}\right)$, $127.00\left(\mathrm{~d}, J\left({ }^{13} \mathrm{C}-{ }^{31} \mathrm{P}\right)=20.9 \mathrm{~Hz}\right), 126.72,125.73\left(\mathrm{~d}, J\left({ }^{13} \mathrm{C}-{ }^{31} \mathrm{P}\right)=14.6 \mathrm{~Hz}\right), 121.00\left(\mathrm{~d}, J\left({ }^{13} \mathrm{C}-\right.\right.$ $\left.\left.{ }^{31} \mathrm{P}\right)=43.9 \mathrm{~Hz}\right), 47.80\left(\mathrm{~d}, J\left({ }^{13} \mathrm{C}-{ }^{31} \mathrm{P}\right)=8.4 \mathrm{~Hz}\right), 24.00\left(\mathrm{~d}, J\left({ }^{13} \mathrm{C}_{-}{ }^{31} \mathrm{P}\right)=4.2 \mathrm{~Hz}\right), 23.67\left(\mathrm{~d}, J\left({ }^{13} \mathrm{C}-\right.\right.$ 
$\left.\left.{ }^{31} \mathrm{P}\right)=4.2 \mathrm{~Hz}\right) ;{ }^{31} \mathrm{P}$ NMR $\left(300 \mathrm{MHz}, \mathrm{CDCl}_{3}\right) \delta 127.39$ (s). Anal. Calcd for $\mathrm{C}_{26} \mathrm{H}_{26} \mathrm{AuClNO}_{2} \mathrm{P}$ : C, 48.20; H, 4.04; N, 2.16. Found: C, 48.15; H, 4.09; N, 2.13.

\section{Gold(I)-complex (46)}

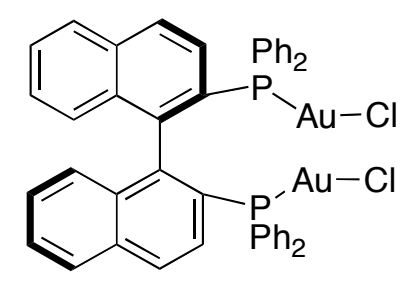

White solid: $\mathrm{mp}>200{ }^{\circ} \mathrm{C}(\mathrm{dec}) ;[\alpha]^{25}=-16\left(\mathrm{c}=0.20, \mathrm{CHCl}_{3}\right) ;{ }^{1} \mathrm{H} \mathrm{NMR}\left(300 \mathrm{MHz}, \mathrm{CDCl}_{3}\right) \delta$ $8.20(\mathrm{~d}, J=8.7 \mathrm{~Hz}, 2 \mathrm{H}), 7,96(\mathrm{~d}, J=8.1 \mathrm{~Hz}, 2 \mathrm{H}), 7.26-7.32(\mathrm{~m}, 17 \mathrm{H}), 7.31-7.28(\mathrm{~m}, 7 \mathrm{H})$, $6.87(\mathrm{t}, J=7.9 \mathrm{~Hz}, 2 \mathrm{H}), 6.67(\mathrm{~d}, J=8.5 \mathrm{~Hz}, 2 \mathrm{H}) ;{ }^{13} \mathrm{C} \mathrm{NMR}\left(75 \mathrm{MHz}, \mathrm{CDCl}_{3}\right) \delta 134.73(\mathrm{~d}$, $\left.J\left({ }^{13} \mathrm{C}-{ }^{31} \mathrm{P}\right)=13.7 \mathrm{~Hz}\right), 134.22\left(\mathrm{~d}, J\left({ }^{13} \mathrm{C}_{-}{ }^{31} \mathrm{P}\right)=14.7 \mathrm{~Hz}\right), 133.90\left(\mathrm{~d}, J\left({ }^{13} \mathrm{C}_{-}{ }^{31} \mathrm{P}\right)=10.5 \mathrm{~Hz}\right)$, $132.97,131.40\left(\mathrm{~d}, J\left({ }^{13} \mathrm{C}_{-}{ }^{31} \mathrm{P}\right)=6.3 \mathrm{~Hz}\right), 131.38\left(\mathrm{~d}, J\left({ }^{13} \mathrm{C}_{-}{ }^{31} \mathrm{P}\right)=5.2 \mathrm{~Hz}\right), 130.88,129.90(\mathrm{~d}$, $\left.J\left({ }^{13} \mathrm{C}-{ }^{31} \mathrm{P}\right)=13.6 \mathrm{~Hz}\right), 129.90\left(\mathrm{~d}, J\left({ }^{13} \mathrm{C}-{ }^{31} \mathrm{P}\right)=11.5 \mathrm{~Hz}\right), 128.60\left(\mathrm{~d}, J\left({ }^{13} \mathrm{C}-{ }^{31} \mathrm{P}\right)=21.9 \mathrm{~Hz}\right)$, 128.12, 127.29, $126.92\left(\mathrm{~d}, J\left({ }^{13} \mathrm{C}-{ }^{31} \mathrm{P}\right)=10.5 \mathrm{~Hz}\right), 126.90,126.05$. MALDI-TOF-MS: $m / z$ : $1051.0\left(\mathrm{M}^{+}-\mathrm{Cl}\right)$.

\section{Gold(I)-complex 47}

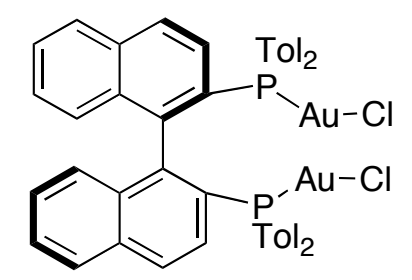

White solid: $\mathrm{mp}>200{ }^{\circ} \mathrm{C}(\mathrm{dec}) ;[\alpha]^{25}=-53.5\left(\mathrm{c}=0.40, \mathrm{CHCl}_{3}\right) ;{ }^{1} \mathrm{H} \mathrm{NMR}\left(300 \mathrm{MHz}, \mathrm{CDCl}_{3}\right)$ $\delta 8.16(\mathrm{~d}, J=8.9 \mathrm{~Hz}, 2 \mathrm{H}), 7.96(\mathrm{~d}, J=8.1 \mathrm{~Hz}, 2 \mathrm{H}), 7.51-7.39(\mathrm{~m}, 8 \mathrm{H}), 7.10-6.96(\mathrm{~m}, 14 \mathrm{H})$, $6.74(\mathrm{~d}, J=8.5 \mathrm{~Hz}, 2 \mathrm{H}), 2.33(\mathrm{~s}, 12 \mathrm{H}) ;{ }^{13} \mathrm{C} \mathrm{NMR}\left(75 \mathrm{MHz}, \mathrm{CDCl}_{3}, \mathrm{DEPT}\right) \delta 141.70(\mathrm{~m}, \mathrm{C})$, $134.61\left(\mathrm{~d}, J\left({ }^{13} \mathrm{C}_{-}{ }^{31} \mathrm{P}\right)=14.1 \mathrm{~Hz}, \mathrm{CH}\right), 133.99(\mathrm{C}), 133.77\left(\mathrm{~d}, J\left({ }^{13} \mathrm{C}_{-}{ }^{31} \mathrm{P}\right)=14.5 \mathrm{~Hz}, \mathrm{CH}\right)$, $129.85(\mathrm{CH}), 129.72(\mathrm{CH}), 129.62(\mathrm{CH}), 129.45\left(\mathrm{~d}, J\left({ }^{13} \mathrm{C}-{ }^{31} \mathrm{P}\right)=1.8 \mathrm{~Hz}, \mathrm{CH}\right), 128.71(\mathrm{CH})$, $128.26(\mathrm{CH}), 128.06(\mathrm{C}), 127.22(\mathrm{C}), 126.85\left(\mathrm{~d}, J\left({ }^{13} \mathrm{C}-{ }^{31} \mathrm{P}\right)=4.2 \mathrm{~Hz}, \mathrm{CH}\right), 126.56(\mathrm{C}), 124.39$ (C), 123.54 (C), $21.33\left(\mathrm{CH}_{3}\right) ;{ }^{31} \mathrm{P} \mathrm{NMR}\left(121.5 \mathrm{MHz}, \mathrm{CDCl}_{3}\right) \delta 22.23$ (s). Anal. Calcd for $\mathrm{C}_{48} \mathrm{H}_{40} \mathrm{Au}_{2} \mathrm{Cl}_{2} \mathrm{P}_{2}$ : C, 50.41; H, 3.53. Found: C, 50.55; H, 3.69.

Chloro [(R)-2-methoxy-2'-diphenylphosphino-1,1'-binaphtyl] gold(I) (48) 


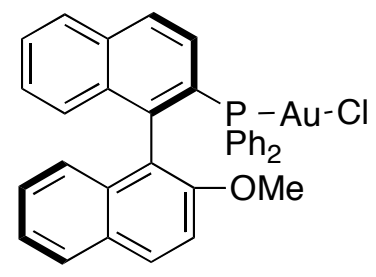

White solid: $\mathrm{mp} 162-165^{\circ} \mathrm{C} ;[\alpha]^{25}=+9.4\left(\mathrm{c}=0.80, \mathrm{CHCl}_{3}\right) ;{ }^{1} \mathrm{H}$ NMR $\left(300 \mathrm{MHz}, \mathrm{CDCl}_{3}\right) \delta$ $8.13(\mathrm{~d}, J=9.1 \mathrm{~Hz}, 1 \mathrm{H}), 7.93(\mathrm{~d}, J=8.5 \mathrm{~Hz}, 2 \mathrm{H}), 7.87$ (d, $J=8.3 \mathrm{~Hz}, 1 \mathrm{H}), 7.57-7.19(\mathrm{~m}$, $16 \mathrm{H}), 6.95(\mathrm{t}, J=8.1 \mathrm{~Hz}, 1 \mathrm{H}), 6.55(\mathrm{~d}, J=8.5 \mathrm{~Hz}, 1 \mathrm{H}), 3.43(\mathrm{~s}, 3 \mathrm{H}) ;{ }^{13} \mathrm{C} \mathrm{NMR}(75 \mathrm{MHz}$, $\left.\mathrm{CDCl}_{3}\right) \delta 154.88,143.89,134.51\left(\right.$ br s), $134.21\left(\mathrm{~d}, J\left({ }^{13} \mathrm{C}^{31} \mathrm{P}\right)=14.6 \mathrm{~Hz}\right), 133.73,133.57(\mathrm{~d}$, $\left.J\left({ }^{13} \mathrm{C}^{31} \mathrm{P}\right)=10.4 \mathrm{~Hz}\right), 131.33,131.18\left(\mathrm{dd}, J\left({ }^{13} \mathrm{C}^{31} \mathrm{P}\right)=6.3,2.1 \mathrm{~Hz}\right), 130.30\left(\mathrm{~d}, J\left({ }^{13} \mathrm{C}^{31} \mathrm{P}\right)=\right.$ $8.4 \mathrm{~Hz}), 129.50\left(\mathrm{~d}, J\left({ }^{13} \mathrm{C}^{31} \mathrm{P}\right)=8.4 \mathrm{~Hz}\right), 128.97,128.85\left(\mathrm{~d}, J\left({ }^{13} \mathrm{C}^{31} \mathrm{P}\right)=3.1 \mathrm{~Hz}\right), 128.78$, $128.70\left(\mathrm{~d}, J\left({ }^{13} \mathrm{C}-{ }^{31} \mathrm{P}\right)=3.1 \mathrm{~Hz}\right), 128.55,128.15\left(\mathrm{~d}, J\left({ }^{13} \mathrm{C}^{31} \mathrm{P}\right)=23.0 \mathrm{~Hz}\right), 128.21\left(\mathrm{~d}, J\left({ }^{13} \mathrm{C}-\right.\right.$ $\left.\left.{ }^{31} \mathrm{P}\right)=8.4 \mathrm{~Hz}\right), 126.90\left(\mathrm{~d}, J\left({ }^{13} \mathrm{C}-{ }^{31} \mathrm{P}\right)=37.6 \mathrm{~Hz}\right), 118.77\left(\mathrm{~d}, J\left({ }^{13} \mathrm{C}-{ }^{31} \mathrm{P}\right)=8.4 \mathrm{~Hz}\right), 112.85$, 55.16. ${ }^{31} \mathrm{P}$ NMR (300 MHz, $\left.\mathrm{CDCl}_{3}\right) \delta 25.26$ (s). HRMS-TOF-ES-MS Calcd for $\mathrm{C}_{33} \mathrm{H}_{25} \mathrm{AuClOP} \cdot \mathrm{Na}$ : $723.0895\left(\mathrm{M}^{+}\right)$. Found: 723.0919. Anal. Calcd for $\mathrm{C}_{33} \mathrm{H}_{25} \mathrm{AuClOP} \cdot \mathrm{H}_{2} \mathrm{O}$ : C, 55.13; H, 3.79. Found: C, 55.21; H, 3.91.

\section{Gold(I)-complex (49)}

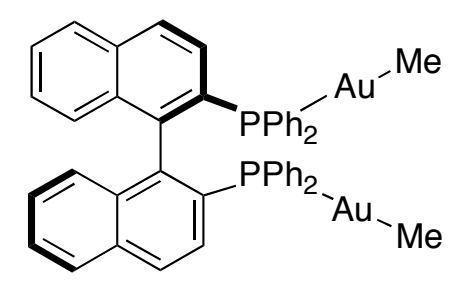

Vitreous solid; $[\alpha]^{25}=+10\left(\mathrm{c}=0.10, \mathrm{CHCl}_{3}\right) ;{ }^{1} \mathrm{H} \mathrm{NMR}\left(300 \mathrm{MHz}, \mathrm{CDCl}_{3}\right) \delta 8.00(\mathrm{~d}, J=$ $8.49 \mathrm{~Hz}, 1 \mathrm{H}), 7.85(\mathrm{~d}, J=8.09 \mathrm{~Hz}, 1 \mathrm{H}), 7.69(\mathrm{dd}, J=11.72,1.72 \mathrm{~Hz}, 1 \mathrm{H}), 7.65(\mathrm{~d}, J=11.73$, $2.03 \mathrm{~Hz}, 1 \mathrm{H}), 7.45(\mathrm{dd}, J=8.49,7.78 \mathrm{~Hz}, 1 \mathrm{H}), 7.37-7.16(\mathrm{~m}, 24 \mathrm{H}), 6.74-6.62(\mathrm{~m}, 3 \mathrm{H}),-0.18$ $(\mathrm{d}, J=7.9 \mathrm{~Hz}, 6 \mathrm{H}) ;{ }^{13} \mathrm{C}$ NMR $\left(75 \mathrm{MHz}, \mathrm{CDCl}_{3}\right) \delta 134.70\left(\mathrm{~d}, J\left({ }^{13} \mathrm{C}^{31} \mathrm{P}\right)=11.5 \mathrm{~Hz}\right), 134.57$ $\left(\mathrm{d}, J\left({ }^{13} \mathrm{C}^{31} \mathrm{P}\right)=10.5 \mathrm{~Hz}\right), 133.20,132.58,131.08,130.20\left(\mathrm{~d}, J\left({ }^{13} \mathrm{C}^{31} \mathrm{P}\right)=11.5 \mathrm{~Hz}\right), 128.80(\mathrm{~d}$, $\left.J\left({ }^{13} \mathrm{C}^{31} \mathrm{P}\right)=6.3 \mathrm{~Hz}\right), 128.54\left(\mathrm{~d}, J\left({ }^{13} \mathrm{C}-{ }^{31} \mathrm{P}\right)=4.2 \mathrm{~Hz}\right), 128.39\left(\mathrm{~d}, J\left({ }^{13} \mathrm{C}_{-}{ }^{31} \mathrm{P}\right)=4.2 \mathrm{~Hz}\right), 127.95$, 127.55, 127.35, 125.93, $8.00\left(\mathrm{~d}, J\left({ }^{13} \mathrm{C}^{31} \mathrm{P}\right)=96.23 \mathrm{~Hz}\right)$. HRMS-TOF-ES-MS Calcd for $\mathrm{C}_{34} \mathrm{H}_{28} \mathrm{Au}_{2} \mathrm{P}_{2}: 861.1260\left(\mathrm{M}^{+}\right)$. Found: 861.1250.

\section{Gold(I)-complex (50)}




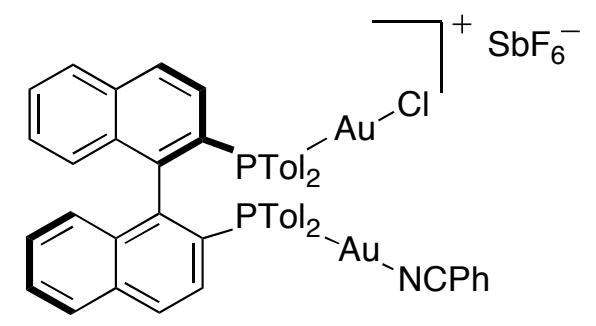

White solid: ${ }^{1} \mathrm{H}$ NMR $\left(300 \mathrm{MHz}, \mathrm{CDCl}_{3}\right) \delta 7.92(\mathrm{~d}, J=9.1 \mathrm{~Hz}, 2 \mathrm{H}), 7.74(\mathrm{~d}, J=8.3 \mathrm{~Hz}, 2 \mathrm{H})$, 7.68-7.59 (m, 5H), 7.50-7.35 (m, 6H), 7.25-7.22 (m, 7H), 6.86-6.79 (m, 6H), $6.65(\mathrm{~d}, J=7.9$ $\mathrm{Hz}, 4 \mathrm{H}), 6.52(\mathrm{~d}, J=8.1 \mathrm{~Hz}, 1 \mathrm{H}), 2.39$ (s, 6H), $2.19(\mathrm{~s}, 6 \mathrm{H}) ;{ }^{31} \mathrm{P} \mathrm{NMR}\left(121.5 \mathrm{MHz}, \mathrm{CDCl}_{3}\right) \delta$ 27.93 (br m). 


\section{X-ray Structure of Complex 39}

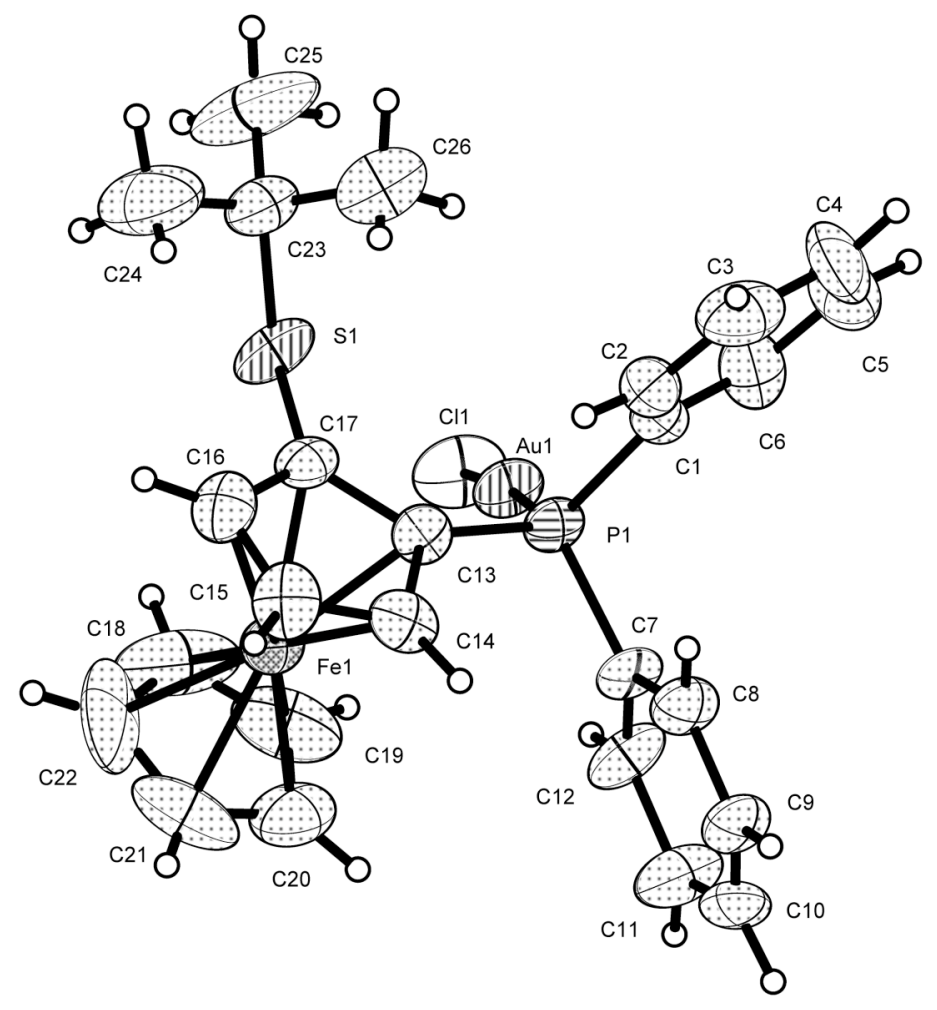

Yellow crystals of $\mathbf{3 9}$ suitable for X-ray diffraction studies were obtained by slow diffusion of hexane vapour into a $\mathrm{CHCl}_{3}$ solution at $4^{\circ} \mathrm{C}$. Crystals were mounted on a glass fibre and transferred to a Bruker Smart 6K CCD area-detector-three-circle diffractometer with a MAC Science Co., LTd. Rotating Anode $\left(\mathrm{CuK}_{\alpha} \lambda=1.54178 \AA\right)$ generator equipped with Goebel mirrors at settings of $50 \mathrm{kV}$ and $100 \mathrm{~mA}^{3}$ X-ray data were collected at $296 \mathrm{~K}$, with a combination of seven runs at different $\varphi$ and $2 \theta$ angles, 3600 frames. The data were collected using $0.3^{\circ}$ wide $\omega$ scans $\left(3 \mathrm{sec} . /\right.$ frame at $2 \theta=50^{\circ}$ and $8 \mathrm{sec}$. $/$ frame at $2 \theta=100^{\circ}$ ), crystal-todetector distance of $4.0 \mathrm{~cm}$.

The substantial redundancy in data allows empirical absorption corrections (SADABS) ${ }^{4}$ to be applied using multiple measurements of symmetry-equivalent reflections. The raw intensity data frames were integrated with the SAINT program, ${ }^{5}$ which also applied corrections for Lorentz and polarization effects. The software package SHELXTL version 6.10 was used for 
space group determination structure solution and refinement. ${ }^{6}$ The structure was solved by direct methods (SHEXS-97), ${ }^{7}$ completed with difference Fourier syntheses, and refined with full-matrix-least-squares using SHELXL- $97^{7}$ minimizing $\omega\left(\mathrm{F}_{0}{ }^{2}-\mathrm{F}_{\mathrm{c}}{ }^{2}\right)^{2}$. Weighted $\mathrm{R}$ factors $\left(\mathrm{R}_{\mathrm{w}}\right)$ and all goodness of fit $\mathrm{S}$ are based on $\mathrm{F}^{2}$; conventional $\mathrm{R}$ factors $(\mathrm{R})$ are based on $\mathrm{F}$. All non-hydrogen atoms were refined with anisotropic displacement parameters. All scattering factors and anomalous dispersions factors are contained in the SHELXTL 6.10 program library. The collection parameters, crystal and data determination data are shown in Table 1.

Table 1. Parameters.

\begin{tabular}{|l|l|}
\hline Diffractometer & Bruker-Siemens Smart CCD \\
\hline Radiation & $\mathrm{CuK}_{\alpha} \lambda=1.54178 \AA$ \\
\hline $\mathrm{T}(\mathrm{K})$ & 296 \\
\hline$\theta\left(^{\mathrm{o}}\right)$ & $4.08-66.57$ \\
\hline Crystal degradation & inappreciable \\
\hline Index Ranges & $-13<=\mathrm{h}<=13,-11<=\mathrm{k}<=16,-20<=1<=14$ \\
\hline
\end{tabular}

Table 2. Crystal data.

\begin{tabular}{|l|l|}
\hline Formula & $\mathrm{C}_{26} \mathrm{H}_{27} \mathrm{AuClFePS}$ \\
\hline Molecular weight & 690.77 \\
\hline Crystal dimensions & $0.30 \times 0.17 \times 0.07 \mathrm{~mm}^{3}$ \\
\hline Crystal system & Orthorhombic \\
\hline Symmetry Group & $\mathrm{P} 2{ }_{1} 2_{1} 2_{1}$ \\
\hline a, $(\AA)$ & $11.04960(10)$ \\
\hline
\end{tabular}




\begin{tabular}{|l|l|}
\hline $\mathrm{b},(\AA)$ & $14.0729(3)$ \\
\hline $\mathrm{c},(\AA)$ & $16.9897(3)$ \\
\hline$\alpha,\left(^{\circ}\right)$ & $90^{\circ}$. \\
\hline$\beta,\left({ }^{\circ}\right)$ & $90^{\circ}$ \\
\hline$\gamma,\left(^{\circ}\right)$ & $90^{\circ}$ \\
\hline Volumen $\left(\AA^{3}\right)$ & $2641.90(8)$ \\
\hline $\mathrm{Z}$ & 4 \\
\hline Calculated density $\left(\mathrm{Mg} / \mathrm{m}^{3}\right)$ & 1.737 \\
\hline F $(000)$ & 1344 \\
\hline$\mu\left(\mathrm{mm}^{-1}\right)$ & 17.010 \\
\hline
\end{tabular}

Table 3. Determination data and goodness of fit.

\begin{tabular}{|l|l|}
\hline Number of reflections observed & 10201 \\
\hline Number of independent reflections & 4021 \\
\hline$\Delta \rho\left(\mathrm{e} \AA^{-3}\right)$ max. and min. & $1.785,-4.566$ \\
\hline $\mathrm{R}$ factors & $\mathrm{R} 1=0.0555, \mathrm{wR} 2=0.1337$ \\
\hline Final $\mathrm{R}[\mathrm{I}>2 \sigma(\mathrm{I})]$ & $\mathrm{R} 1=0.0541, \mathrm{wR} 2=0.1321$ \\
\hline
\end{tabular}

Table 4. Atomic coordinates $\left(\mathrm{x} 10^{4}\right)$ and equivalent isotropic displacement parameters $\left(\AA^{2} \mathrm{x}\right.$ $10^{3}$ ) for 39. $U(\mathrm{eq})$ is defined as one third of the trace of the orthogonalized Uij tensor.

$\begin{array}{llll}\mathrm{x} & \mathrm{y} & \mathrm{z} & \mathrm{U}(\mathrm{eq})\end{array}$ 


\begin{tabular}{|c|c|c|c|c|}
\hline $\mathrm{Au}(1)$ & $9350(1)$ & $5937(1)$ & $6640(1)$ & $42(1)$ \\
\hline $\mathrm{P}(1)$ & $7596(2)$ & $6003(2)$ & $7286(1)$ & $34(1)$ \\
\hline $\mathrm{Cl}(1)$ & $11054(2)$ & $5945(3)$ & $5873(2)$ & $64(1)$ \\
\hline$C(7)$ & $6319(7)$ & $5955(8)$ & $6611(5)$ & $36(2)$ \\
\hline$C(10)$ & $4355(9)$ & $5970(9)$ & $5595(7)$ & $53(3)$ \\
\hline$C(12)$ & $6517(9)$ & $6096(11)$ & $5828(6)$ & $53(3)$ \\
\hline$C(11)$ & $5510(11)$ & $6123(12)$ & $5304(7)$ & $66(4)$ \\
\hline $\mathrm{Fe}(1)$ & $7548(1)$ & $8410(1)$ & $7340(1)$ & $40(1)$ \\
\hline$C(14)$ & $6251(9)$ & $7609(9)$ & $7884(6)$ & $44(3)$ \\
\hline$C(13)$ & $7366(8)$ & $7081(8)$ & $7822(6)$ & $36(2)$ \\
\hline$C(15)$ & $6471(10)$ & $8429(10)$ & $8326(6)$ & $52(3)$ \\
\hline$C(3)$ & $6342(13)$ & $4413(14)$ & $9127(8)$ & $76(5)$ \\
\hline$C(2)$ & $6641(10)$ & $5187(10)$ & $8662(6)$ & $46(3)$ \\
\hline$C(8)$ & $5152(9)$ & $5824(9)$ & $6890(6)$ & $46(3)$ \\
\hline$C(9)$ & $4154(9)$ & $5849(10)$ & $6376(7)$ & $53(3)$ \\
\hline$C(1)$ & $7299(8)$ & $5046(8)$ & $7964(7)$ & $39(3)$ \\
\hline$C(19)$ & $8222(17)$ & $8317(15)$ & $6222(8)$ & $84(6)$ \\
\hline$C(17)$ & $8288(9)$ & $7652(8)$ & $8233(5)$ & $38(2)$ \\
\hline$C(18)$ & $8913(14)$ & $8900(20)$ & $6676(10)$ & $108(9)$ \\
\hline$C(21)$ & $7024(18)$ & $9499(14)$ & $6621(10)$ & $88(6)$ \\
\hline$C(16)$ & $7707(11)$ & $8485(10)$ & $8532(6)$ & $48(3)$ \\
\hline$C(4)$ & $6757(15)$ & $3482(12)$ & $8931(11)$ & $76(4)$ \\
\hline $\mathrm{S}(1)$ & $9841(2)$ & $7383(3)$ & $8277(2)$ & $56(1)$ \\
\hline$C(6)$ & $7696(11)$ & $4160(11)$ & $7780(8)$ & $60(3)$ \\
\hline
\end{tabular}




$\begin{array}{lllll}\mathrm{C}(22) & 8180(30) & 9669(17) & 6942(10) & 130(11) \\ \mathrm{C}(5) & 7412(16) & 3359(12) & 8258(12) & 86(5) \\ \mathrm{C}(20) & 7086(14) & 8667(13) & 6184(7) & 72(5) \\ \mathrm{C}(23) & 10120(12) & 7147(12) & 9325(7) & 59(3) \\ \mathrm{C}(24) & 10040(20) & 8004(14) & 9816(9) & 106(7) \\ \mathrm{C}(25) & 11408(17) & 6759(18) & 9326(11) & 125(10) \\ \mathrm{C}(26) & 9240(20) & 6362(17) & 9615(14) & 139(10)\end{array}$

Table 5. Bond lengths $[\AA]$ and angles $\left[^{\circ}\right]$ for 39.

$\begin{array}{llll}\mathrm{Au}(1)-\mathrm{P}(1) & 2.230(2) & \mathrm{Fe}(1)-\mathrm{C}(21) & 2.044(14) \\ \mathrm{Au}(1)-\mathrm{Cl}(1) & 2.289(2) & \mathrm{Fe}(1)-\mathrm{C}(19) & 2.045(13) \\ \mathrm{P}(1)-\mathrm{C}(13) & 1.787(12) & \mathrm{Fe}(1)-\mathrm{C}(13) & 2.052(12) \\ \mathrm{P}(1)-\mathrm{C}(1) & 1.802(11) & \mathrm{Fe}(1)-\mathrm{C}(15) & 2.056(10) \\ \mathrm{P}(1)-\mathrm{C}(7) & 1.820(8) & \mathrm{Fe}(1)-\mathrm{C}(20) & 2.061(12) \\ \mathrm{C}(7)-\mathrm{C}(12) & 1.362(14) & \mathrm{C}(14)-\mathrm{C}(15) & 1.397(18) \\ \mathrm{C}(7)-\mathrm{C}(8) & 1.387(14) & \mathrm{C}(14)-\mathrm{C}(13) & 1.443(14) \\ \mathrm{C}(10)-\mathrm{C}(9) & 1.356(17) & \mathrm{C}(13)-\mathrm{C}(17) & 1.474(14) \\ \mathrm{C}(10)-\mathrm{C}(11) & 1.385(17) & \mathrm{C}(15)-\mathrm{C}(16) & 1.413(17) \\ \mathrm{C}(12)-\mathrm{C}(11) & 1.425(14) & \mathrm{C}(3)-\mathrm{C}(2) & 1.385(19) \\ \mathrm{Fe}(1)-\mathrm{C}(18) & 2.007(15) & \mathrm{C}(3)-\mathrm{C}(4) & 1.43(2) \\ \mathrm{Fe}(1)-\mathrm{C}(22) & 2.021(17) & \mathrm{C}(2)-\mathrm{C}(1) & 1.404(16) \\ \mathrm{Fe}(1)-\mathrm{C}(17) & 2.028(9) & \mathrm{C}(8)-\mathrm{C}(9) & 1.407(14) \\ \mathrm{Fe}(1)-\mathrm{C}(16) & 2.036(10) & \mathrm{C}(1)-\mathrm{C}(6) & 1.359(18) \\ \mathrm{Fe}(1)-\mathrm{C}(14) & 2.044(11) & \mathrm{C}(19)-\mathrm{C}(20) & 1.35(2)\end{array}$




\begin{tabular}{|c|c|c|c|c|}
\hline $\mathrm{C}(19)-\mathrm{C}(18)$ & 1.36( & & $\mathrm{C}(18)-\mathrm{Fe}(1)-\mathrm{C}(22)$ & $41.3(10)$ \\
\hline$C(17)-C(16)$ & 1.430 & (17) & $\mathrm{C}(18)-\mathrm{Fe}(1)-\mathrm{C}(17)$ & $107.4(5)$ \\
\hline $\mathrm{C}(17)-\mathrm{S}(1)$ & 1.758 & (10) & $\mathrm{C}(22)-\mathrm{Fe}(1)-\mathrm{C}(17)$ & $124.9(9)$ \\
\hline $\mathrm{C}(18)-\mathrm{C}(22)$ & 1.42( & & $\mathrm{C}(18)-\mathrm{Fe}(1)-\mathrm{C}(16)$ & $118.4(7)$ \\
\hline$C(21)-C(20)$ & 1.39( & & $\mathrm{C}(22)-\mathrm{Fe}(1)-\mathrm{C}(16)$ & $104.9(7)$ \\
\hline$C(21)-C(22)$ & 1.41( & & $\mathrm{C}(17)-\mathrm{Fe}(1)-\mathrm{C}(16)$ & $41.2(5)$ \\
\hline$C(4)-C(5)$ & 1.36( & & $\mathrm{C}(18)-\mathrm{Fe}(1)-\mathrm{C}(14)$ & $166.4(9)$ \\
\hline$S(1)-C(23)$ & 1.839 & (12) & $\mathrm{C}(22)-\mathrm{Fe}(1)-\mathrm{C}(14)$ & $151.3(11)$ \\
\hline$C(6)-C(5)$ & 1.42( & & $\mathrm{C}(17)-\mathrm{Fe}(1)-\mathrm{C}(14)$ & $69.8(4)$ \\
\hline$C(23)-C(24)$ & 1.47( & & $\mathrm{C}(16)-\mathrm{Fe}(1)-\mathrm{C}(14)$ & $68.9(5)$ \\
\hline$C(23)-C(25)$ & 1.52( & & $\mathrm{C}(18)-\mathrm{Fe}(1)-\mathrm{C}(21)$ & $67.5(7)$ \\
\hline$C(23)-C(26)$ & 1.55( & & $\mathrm{C}(22)-\mathrm{Fe}(1)-\mathrm{C}(21)$ & $40.6(9)$ \\
\hline $\mathrm{P}(1)-\mathrm{Au}(1)-\mathrm{Cl}($ & (1) & $174.25(10)$ & $\mathrm{C}(17)-\mathrm{Fe}(1)-\mathrm{C}(21)$ & $162.9(8)$ \\
\hline $\mathrm{C}(13)-\mathrm{P}(1)-\mathrm{C}(1$ & & $106.4(5)$ & $\mathrm{C}(16)-\mathrm{Fe}(1)-\mathrm{C}(21)$ & $125.4(7)$ \\
\hline $\mathrm{C}(13)-\mathrm{P}(1)-\mathrm{C}(7$ & & $104.0(5)$ & $\mathrm{C}(14)-\mathrm{Fe}(1)-\mathrm{C}(21)$ & $119.0(6)$ \\
\hline $\mathrm{C}(1)-\mathrm{P}(1)-\mathrm{C}(7)$ & & $103.6(5)$ & $\mathrm{C}(18)-\mathrm{Fe}(1)-\mathrm{C}(19)$ & $39.3(8)$ \\
\hline$C(13)-P(1)-A u$ & $\mathrm{u}(1)$ & $114.1(3)$ & $\mathrm{C}(22)-\mathrm{Fe}(1)-\mathrm{C}(19)$ & $67.6(9)$ \\
\hline $\mathrm{C}(1)-\mathrm{P}(1)-\mathrm{Au}($ & & $116.3(3)$ & $\mathrm{C}(17)-\mathrm{Fe}(1)-\mathrm{C}(19)$ & $121.0(6)$ \\
\hline $\mathrm{C}(7)-\mathrm{P}(1)-\mathrm{Au}($ & & $111.2(3)$ & $C(16)-F e(1)-C(19)$ & $153.7(7)$ \\
\hline$C(12)-C(7)-C($ & & $120.2(8)$ & $\mathrm{C}(14)-\mathrm{Fe}(1)-\mathrm{C}(19)$ & $129.9(7)$ \\
\hline$C(12)-C(7)-P(1$ & & 119.1(6) & $\mathrm{C}(21)-\mathrm{Fe}(1)-\mathrm{C}(19)$ & $66.1(7)$ \\
\hline $\mathrm{C}(8)-\mathrm{C}(7)-\mathrm{P}(1)$ & & $120.6(7)$ & $\mathrm{C}(18)-\mathrm{Fe}(1)-\mathrm{C}(13)$ & $127.8(8)$ \\
\hline$C(9)-C(10)-C(1$ & (11) & $121.3(9)$ & $\mathrm{C}(22)-\mathrm{Fe}(1)-\mathrm{C}(13)$ & $165.1(11)$ \\
\hline$C(7)-C(12)-C($ & (11) & 119.2(9) & $\mathrm{C}(17)-\mathrm{Fe}(1)-\mathrm{C}(13)$ & $42.4(4)$ \\
\hline$C(10)-C(11)-C$ & $C(12)$ & $119.5(10)$ & $\mathrm{C}(16)-\mathrm{Fe}(1)-\mathrm{C}(13)$ & $70.1(5)$ \\
\hline
\end{tabular}




\begin{tabular}{|c|c|c|c|}
\hline $\mathrm{C}(14)-\mathrm{Fe}(1)-\mathrm{C}(13)$ & $41.3(4)$ & $\mathrm{C}(17)-\mathrm{C}(13)-\mathrm{P}(1)$ & $127.3(7)$ \\
\hline $\mathrm{C}(21)-\mathrm{Fe}(1)-\mathrm{C}(13)$ & $153.5(7)$ & $\mathrm{C}(14)-\mathrm{C}(13)-\mathrm{Fe}(1)$ & $69.1(6)$ \\
\hline$C(19)-\mathrm{Fe}(1)-\mathrm{C}(13)$ & $110.4(6)$ & $\mathrm{C}(17)-\mathrm{C}(13)-\mathrm{Fe}(1)$ & $68.0(6)$ \\
\hline $\mathrm{C}(18)-\mathrm{Fe}(1)-\mathrm{C}(15)$ & $152.7(8)$ & $\mathrm{P}(1)-\mathrm{C}(13)-\mathrm{Fe}(1)$ & $123.8(5)$ \\
\hline $\mathrm{C}(22)-\mathrm{Fe}(1)-\mathrm{C}(15)$ & $117.5(9)$ & $\mathrm{C}(14)-\mathrm{C}(15)-\mathrm{C}(16)$ & $110.4(11)$ \\
\hline $\mathrm{C}(17)-\mathrm{Fe}(1)-\mathrm{C}(15)$ & $68.3(4)$ & $\mathrm{C}(14)-\mathrm{C}(15)-\mathrm{Fe}(1)$ & $69.6(6)$ \\
\hline$C(16)-\mathrm{Fe}(1)-\mathrm{C}(15)$ & $40.4(5)$ & $\mathrm{C}(16)-\mathrm{C}(15)-\mathrm{Fe}(1)$ & $69.1(6)$ \\
\hline $\mathrm{C}(14)-\mathrm{Fe}(1)-\mathrm{C}(15)$ & $39.9(5)$ & $C(2)-C(3)-C(4)$ & $120.8(14)$ \\
\hline $\mathrm{C}(21)-\mathrm{Fe}(1)-\mathrm{C}(15)$ & $108.3(6)$ & $\mathrm{C}(3)-\mathrm{C}(2)-\mathrm{C}(1)$ & $119.6(14)$ \\
\hline $\mathrm{C}(19)-\mathrm{Fe}(1)-\mathrm{C}(15)$ & $165.7(7)$ & $C(7)-C(8)-C(9)$ & $120.8(10)$ \\
\hline $\mathrm{C}(13)-\mathrm{Fe}(1)-\mathrm{C}(15)$ & $68.3(5)$ & $C(10)-C(9)-C(8)$ & $118.8(10)$ \\
\hline $\mathrm{C}(18)-\mathrm{Fe}(1)-\mathrm{C}(20)$ & $65.8(6)$ & $C(6)-C(1)-C(2)$ & $119.4(11)$ \\
\hline $\mathrm{C}(22)-\mathrm{Fe}(1)-\mathrm{C}(20)$ & $67.2(7)$ & $C(6)-C(1)-P(1)$ & $118.6(9)$ \\
\hline $\mathrm{C}(17)-\mathrm{Fe}(1)-\mathrm{C}(20)$ & $154.9(6)$ & $\mathrm{C}(2)-\mathrm{C}(1)-\mathrm{P}(1)$ & $121.9(9)$ \\
\hline $\mathrm{C}(16)-\mathrm{Fe}(1)-\mathrm{C}(20)$ & $163.8(7)$ & $\mathrm{C}(20)-\mathrm{C}(19)-\mathrm{C}(18)$ & 109.1(18) \\
\hline $\mathrm{C}(14)-\mathrm{Fe}(1)-\mathrm{C}(20)$ & $110.8(5)$ & $\mathrm{C}(20)-\mathrm{C}(19)-\mathrm{Fe}(1)$ & $71.5(8)$ \\
\hline $\mathrm{C}(21)-\mathrm{Fe}(1)-\mathrm{C}(20)$ & $39.5(7)$ & $\mathrm{C}(18)-\mathrm{C}(19)-\mathrm{Fe}(1)$ & $68.9(9)$ \\
\hline $\mathrm{C}(19)-\mathrm{Fe}(1)-\mathrm{C}(20)$ & $38.4(7)$ & $C(16)-C(17)-C(13)$ & $107.8(9)$ \\
\hline $\mathrm{C}(13)-\mathrm{Fe}(1)-\mathrm{C}(20)$ & $121.1(6)$ & $C(16)-C(17)-S(1)$ & $126.8(8)$ \\
\hline $\mathrm{C}(15)-\mathrm{Fe}(1)-\mathrm{C}(20)$ & $129.1(6)$ & $\mathrm{C}(13)-\mathrm{C}(17)-\mathrm{S}(1)$ & $125.2(8)$ \\
\hline$C(15)-C(14)-C(13)$ & $108.5(10)$ & $\mathrm{C}(16)-\mathrm{C}(17)-\mathrm{Fe}(1)$ & $69.7(6)$ \\
\hline $\mathrm{C}(15)-\mathrm{C}(14)-\mathrm{Fe}(1)$ & $70.5(7)$ & $\mathrm{C}(13)-\mathrm{C}(17)-\mathrm{Fe}(1)$ & $69.7(5)$ \\
\hline $\mathrm{C}(13)-\mathrm{C}(14)-\mathrm{Fe}(1)$ & $69.7(6)$ & $\mathrm{S}(1)-\mathrm{C}(17)-\mathrm{Fe}(1)$ & $122.6(5)$ \\
\hline$C(14)-C(13)-C(17)$ & $105.9(10)$ & $C(19)-C(18)-C(22)$ & $108.7(17)$ \\
\hline $\mathrm{C}(14)-\mathrm{C}(13)-\mathrm{P}(1)$ & $126.6(8)$ & $\mathrm{C}(19)-\mathrm{C}(18)-\mathrm{Fe}(1)$ & $71.8(9)$ \\
\hline
\end{tabular}




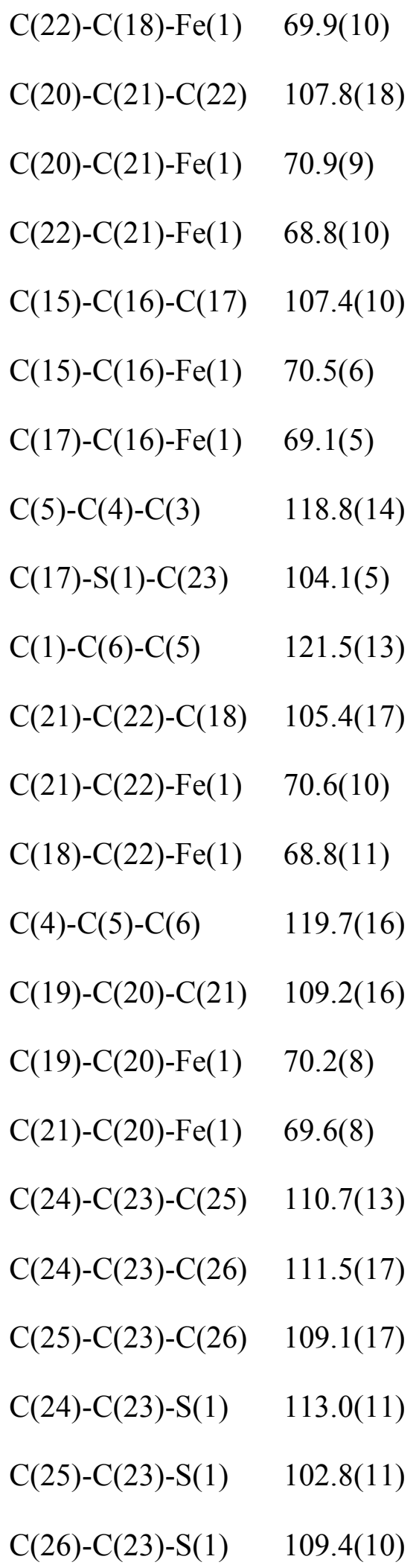


Table 6. Anisotropic displacement parameters $\left(\AA^{2} \times 10^{3}\right)$ for 39. The anisotropic displacement factor exponent takes the form: $-2 \pi^{2}\left[\left(h^{*}\right)^{2} U_{11}+\ldots+2 h k a+b+U_{12}\right]$

$\mathrm{U}^{11} \quad \mathrm{U}^{22} \quad \mathrm{U}^{33} \quad \mathrm{U}^{23} \quad \mathrm{U}^{13} \quad \mathrm{U}^{12}$

$\begin{array}{lllllll}\mathrm{Au}(1) & 18(1) & 59(1) & 48(1) & 0(1) & 1(1) & 3(1) \\ \mathrm{P}(1) & 21(1) & 45(2) & 37(1) & 2(1) & 1(1) & -2(1) \\ \mathrm{Cl}(1) & 23(1) & 104(3) & 66(1) & 6(2) & 13(1) & 8(1) \\ \mathrm{C}(7) & 6(3) & 54(7) & 49(5) & 3(6) & -4(3) & 2(3) \\ \mathrm{C}(10) & 35(5) & 56(8) & 68(6) & 18(6) & -20(5) & 4(6) \\ \mathrm{C}(12) & 26(4) & 85(11) & 46(5) & -13(6) & -6(4) & -3(5) \\ \mathrm{C}(11) & 47(6) & 106(13) & 46(5) & 10(7) & -10(5) & -10(7) \\ \mathrm{Fe}(1) & 33(1) & 48(1) & 40(1) & 7(1) & 2(1) & -4(1) \\ \mathrm{C}(14) & 31(5) & 51(8) & 50(5) & 15(5) & 10(4) & 0(5) \\ \mathrm{C}(13) & 20(4) & 41(7) & 47(5) & 2(5) & 5(3) & 1(4) \\ \mathrm{C}(15) & 56(6) & 55(8) & 45(5) & -9(6) & 16(5) & -1(5) \\ \mathrm{C}(3) & 67(8) & 108(16) & 53(6) & 32(8) & -12(6) & -23(8) \\ \mathrm{C}(2) & 50(6) & 45(8) & 45(5) & 8(5) & -5(4) & -8(5) \\ \mathrm{C}(8) & 29(5) & 58(9) & 53(5) & 9(6) & -5(4) & -3(5) \\ \mathrm{C}(9) & 26(5) & 65(9) & 67(6) & 14(6) & -13(4) & -12(5) \\ \mathrm{C}(1) & 19(4) & 40(7) & 59(5) & 13(5) & -7(4) & -3(4) \\ \mathrm{C}(19) & 88(12) & 109(15) & 56(7) & 33(9) & 30(8) & 31(11) \\ \mathrm{C}(17) & 39(5) & 49(7) & 25(4) & 7(4) & -2(3) & -5(4) \\ \mathrm{C}(18) & 44(8) & 200(30) & 77(10) & 69(16) & 0(7) & -9(11) \\ \mathrm{C}(16) & 56(6) & 54(9) & 35(5) & -6(5) & 6(4) & -7(5)\end{array}$




$\begin{array}{lllllll}\mathrm{C}(4) & 79(10) & 39(10) & 110(12) & 26(9) & -3(9) & -12(7) \\ \mathrm{S}(1) & 31(1) & 89(3) & 47(1) & -10(2) & -8(1) & -3(1) \\ \mathrm{C}(6) & 56(7) & 49(10) & 77(7) & -2(7) & 5(6) & -1(6) \\ \mathrm{C}(22) & 240(30) & 79(16) & 69(10) & 28(11) & -6(16) & -101(19) \\ \mathrm{C}(5) & 81(10) & 50(11) & 128(15) & 11(11) & -27(11) & 7(8) \\ \mathrm{C}(20) & 73(9) & 96(14) & 47(6) & 22(8) & -13(6) & -14(8) \\ \mathrm{C}(23) & 61(7) & 66(10) & 51(6) & -3(7) & -22(5) & 0(7) \\ \mathrm{C}(24) & 130(16) & 117(18) & 71(9) & -26(10) & -45(10) & 40(13) \\ \mathrm{C}(25) & 84(11) & 190(30) & 97(11) & -60(14) & -53(10) & 71(14) \\ \mathrm{C}(26) & 170(30) & 113(19) & 138(18) & 11(16) & -96(19) & -22(17)\end{array}$

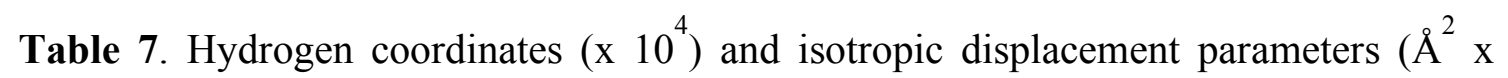
$10^{3}$ ) for 39 .

\begin{tabular}{lllll}
\hline & & & & \\
& & & $\mathrm{U}(\mathrm{eq})$ \\
$\mathrm{H}(10)$ & 3705 & 5951 & 5248 & 63 \\
$\mathrm{H}(12)$ & 7300 & 6173 & 5638 & 63 \\
$\mathrm{H}(11)$ & 5628 & 6242 & 4771 & 80 \\
$\mathrm{H}(14)$ & 5512 & 7434 & 7667 & 53 \\
$\mathrm{H}(15)$ & 5886 & 8873 & 8465 & 62 \\
$\mathrm{H}(3)$ & 5866 & 4502 & 9572 & 91 \\
$\mathrm{H}(2)$ & 6408 & 5796 & 8810 & 56 \\
$\mathrm{H}(8)$ & 5027 & 5718 & 7424 & 56 \\
$\mathrm{H}(9)$ & 3371 & 5783 & 6568 & 63
\end{tabular}




\begin{tabular}{|c|c|c|c|c|}
\hline H(19) & 8490 & 7765 & 5977 & 101 \\
\hline $\mathrm{H}(18)$ & 9728 & 8818 & 6793 & 125 \\
\hline $\mathrm{H}(21)$ & 6342 & 9878 & 6690 & 106 \\
\hline $\mathrm{H}(16)$ & 8076 & 8975 & 8811 & 8 \\
\hline $\mathrm{H}(4)$ & 6583 & 2968 & 9256 & 91 \\
\hline $\mathrm{H}(6)$ & 8165 & 4074 & 7331 & 3 \\
\hline $\mathrm{H}(22)$ & 8414 & 10174 & 7261 & 56 \\
\hline $\mathrm{H}(5)$ & 7671 & 2756 & 8113 & 10 \\
\hline $\mathrm{H}(20)$ & 6446 & 8394 & 5909 & 86 \\
\hline $\mathrm{H}(24 \mathrm{~A})$ & 9201 & 8170 & 9889 & 50 \\
\hline $\mathrm{H}(24 \mathrm{~B})$ & 10449 & 8519 & 9560 & 15 \\
\hline $\mathrm{H}(24 \mathrm{C})$ & 10403 & 7885 & 10318 & 150 \\
\hline $\mathrm{H}(25 \mathrm{~A})$ & 11952 & 7237 & 9133 & 187 \\
\hline $\mathrm{H}(25 \mathrm{~B})$ & 11449 & 6209 & 8992 & 18 \\
\hline $\mathrm{H}(25 \mathrm{C})$ & 11633 & 6586 & 9852 & 18 \\
\hline $\mathrm{H}(26 \mathrm{~A})$ & 9539 & 6102 & 10101 & $20 S$ \\
\hline $\mathrm{H}(26 \mathrm{~B})$ & 9196 & 5867 & 9227 & 20 \\
\hline $\mathrm{H}(26 \mathrm{C})$ & 8455 & 6629 & 9697 & \\
\hline
\end{tabular}




\section{X-ray Structure of Complex 47}

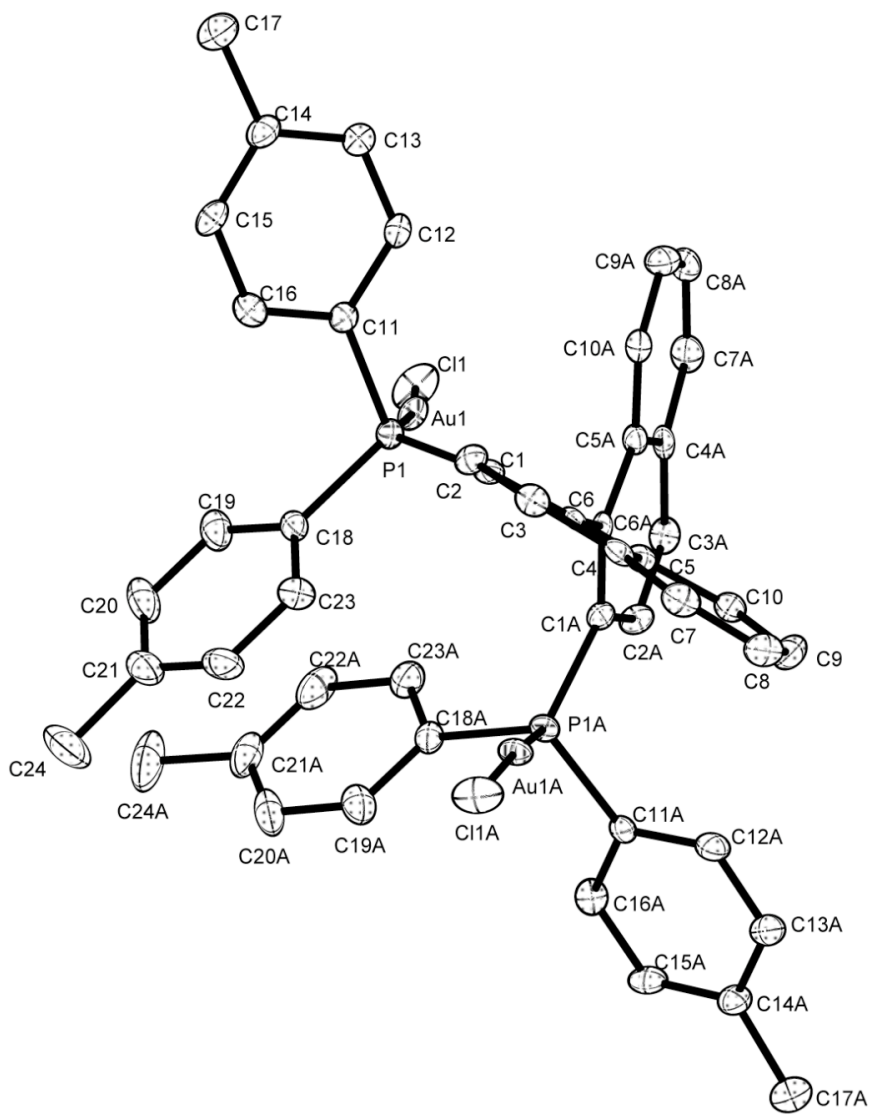

Colorless crystals of $\mathbf{4 7}$ suitable for X-ray diffraction studies were obttained by slow diffusion of hexane vapour into a $\mathrm{CHCl}_{3}$ solution at $4{ }^{\circ} \mathrm{C}$. Crystals were mounted on a glass fiber and transferred to a Bruker Smart 6K CCD area-detector-three-circle diffractometer with a MAC Science Co., LTd. Rotating Anode $\left(\mathrm{CuK}_{\alpha} \lambda=1.54178 \AA\right)$ generator equipped with Goebel mirrors at settings of $50 \mathrm{kV}$ and $100 \mathrm{~mA}$. X-ray data were collected at $100 \mathrm{~K}$, with a combination of seven runs at different $\varphi$ and $2 \theta$ angles, 3600 frames. The data were collected using $0.3^{\circ}$ wide $\omega$ scans $\left(3 \mathrm{sec}\right.$. $/$ frame at $2 \theta=50^{\circ}$ and $5 \mathrm{sec}$./frame at $2 \theta=100^{\circ}$ ), crystal-to-detector distance of $4.0 \mathrm{~cm}$. The collection parameters, crystal and data determination data are shown in Tables 8-14.

Table 8. Parameters. 


\begin{tabular}{|l|l|}
\hline Radiation & $\mathrm{CuK}_{\alpha} \lambda=1.54178 \AA$ \\
\hline $\mathrm{T}(\mathrm{K})$ & 293 \\
\hline$\theta\left(^{\circ}\right)$ & $4.49-70.49$ \\
\hline Crystal degradation & inappreciable \\
\hline Index Ranges & $-13<=\mathrm{h}<=12,-12<=\mathrm{k}<=13,-27<=1<=32$ \\
\hline
\end{tabular}

Table 9. Crystal data.

\begin{tabular}{|l|l|}
\hline Formula & C48 H40 Au2 Cl2 P2 \\
\hline Molecular weight & 1143.57 \\
\hline Crystal dimensions & $0.20 \times 0.09 \times 0.04 \mathrm{~mm}^{3}$ \\
\hline Crystal system & Trigonal \\
\hline Symmetry Group & P3221 \\
\hline a, $(\AA)$ & $11.37890(10)$ \\
\hline b, $(\AA)$ & $11.37890(10)$ \\
\hline c, $(\AA)$ & $27.7151(3)$ \\
\hline$\alpha,\left(^{\circ}\right)$ & $90^{\circ}$. \\
\hline$\beta,\left({ }^{\circ}\right)$ & $90^{\circ}$ \\
\hline$\gamma,\left({ }^{\circ}\right)$ & $120^{\circ}$ \\
\hline Volumen $\left(\AA^{3}\right)$ & $3107.76(5)$ \\
\hline$Z$ & 3 \\
\hline
\end{tabular}




\begin{tabular}{|l|l|}
\hline Calculated density $\left(\mathrm{Mg} / \mathrm{m}^{3}\right)$ & 1.833 \\
\hline $\mathrm{F}(000)$ & 1650 \\
\hline$\mu\left(\mathrm{mm}^{-1}\right)$ & 15.289 \\
\hline
\end{tabular}

Table 10. Determination data and goodness of fit.

\begin{tabular}{|l|l|}
\hline Number of reflections observed & 12054 \\
\hline Number of independent reflections & 3778 \\
\hline$\Delta \rho\left(\mathrm{e} \AA^{-3}\right)$ max. and min. & $0.461,-0.513$ \\
\hline R factors & $\mathrm{R} 1=0.0185, \mathrm{wR} 2=0.0450$ \\
\hline Final R $[\mathrm{I}>2 \sigma(\mathrm{I})]$ & $\mathrm{R} 1=0.0183, \mathrm{wR} 2=0.0448$ \\
\hline
\end{tabular}

Table 11. Atomic coordinates (x $10^{4}$ ) and equivalent isotropic displacement parameters $\left(\AA^{2} \times 10^{3}\right)$ for $47 . \mathrm{U}(\mathrm{eq})$ is defined as one third of the trace of the orthogonalized $U_{\mathrm{ij}}$ tensor.

$\begin{array}{llll}\mathrm{x} & \mathrm{y} & \mathrm{z} & \mathrm{U}(\mathrm{eq})\end{array}$

$\begin{array}{lllll}\mathrm{Au}(1) & 7025(1) & 9022(1) & 3126(1) & 14(1) \\ \mathrm{Cl}(1) & 5725(1) & 8136(1) & 2449(1) & 27(1) \\ \mathrm{P}(1) & 8146(1) & 9623(1) & 3827(1) & 11(1) \\ \mathrm{C}(1) & 9498(4) & 11345(4) & 3950(1) & 13(1) \\ \mathrm{C}(2) & 9904(4) & 11669(4) & 4441(1) & 14(1) \\ \mathrm{C}(3) & 11048(4) & 12843(4) & 4564(1) & 15(1) \\ \mathrm{C}(4) & 11837(4) & 13813(3) & 4206(1) & 14(1)\end{array}$




\begin{tabular}{|c|c|c|c|c|}
\hline$C(5)$ & $11383(4)$ & $13543(4)$ & $3717(1)$ & $12(1)$ \\
\hline$C(6)$ & $216(4)$ & 1228 & 359 & $10(1$ \\
\hline (7) & $5(4)$ & $15003(4)$ & $317(1)$ & $19(1)$ \\
\hline (8) & $3815(4)$ & $15924(4)$ & $3968(1)$ & 21(1) \\
\hline $\mathrm{C}(9)$ & $3344(4)$ & $15711(4)$ & 3487 & $20(1)$ \\
\hline$C(10)$ & $12156(4)$ & $14553(4)$ & $3366(1)$ & $17(1)$ \\
\hline$C(11)$ & $6891(3)$ & $9212(3)$ & $4299(1)$ & $12(1)$ \\
\hline$C(12)$ & $6021(4)$ & 9745 & 4278 & $18(1)$ \\
\hline$C(13)$ & $4948(4)$ & $9318(4)$ & $4594(1)$ & $21(1)$ \\
\hline$C(14)$ & $4697(4)$ & $8328(4)$ & $4947(1)$ & $18(1)$ \\
\hline$C(15)$ & $5595(4)$ & $7846(4)$ & $4978(1)$ & $17(1)$ \\
\hline$C(16)$ & $6672(4)$ & $8262(4)$ & $4659(1)$ & $15(1)$ \\
\hline$C(17)$ & $3467(4)$ & $7786(5)$ & $5269(2)$ & $28(1)$ \\
\hline $\mathrm{C}(18)$ & $8935(4)$ & $8597(4)$ & $3936(1)$ & $15(1)$ \\
\hline$C(19)$ & $8254(4)$ & $7256(4)$ & $3776(1)$ & $23(1)$ \\
\hline$C(20)$ & $8821(5)$ & $6448(4)$ & $3835(1)$ & $29(1)$ \\
\hline$C(21)$ & $10074(5)$ & $6934(5)$ & $4054(2)$ & $30(1)$ \\
\hline$C(22)$ & $10755(5)$ & $8269(5)$ & $4211(2)$ & $29(1)$ \\
\hline$C(23)$ & $10189(4)$ & $9091(4)$ & $4154(1)$ & $22(1$ \\
\hline $\mathrm{C}(24$ & $10722(7)$ & $6067(6)$ & $4109(2)$ & 48( \\
\hline
\end{tabular}

Table 12. Bond lengths $[\AA]$ and angles $\left[^{\circ}\right]$ for 47.

\begin{tabular}{llll}
\hline $\mathrm{Au}(1)-\mathrm{P}(1)$ & $2.2353(8)$ & $\mathrm{P}(1)-\mathrm{C}(18)$ & $1.819(4)$ \\
$\mathrm{Au}(1)-\mathrm{Cl}(1)$ & $2.2880(9)$ & $\mathrm{P}(1)-\mathrm{C}(1)$ & $1.819(4)$ \\
$\mathrm{P}(1)-\mathrm{C}(11)$ & $1.818(3)$ & $\mathrm{C}(1)-\mathrm{C}(6)$ & $1.386(5)$
\end{tabular}




\begin{tabular}{|c|c|c|c|}
\hline $\mathrm{C}(1)-\mathrm{C}(2)$ & $1.426(5)$ & $\mathrm{C}(11)-\mathrm{P}(1)-\mathrm{C}(18)$ & $106.99(16)$ \\
\hline$C(2)-C(3)$ & $1.362(5)$ & $\mathrm{C}(11)-\mathrm{P}(1)-\mathrm{C}(1)$ & $105.92(16)$ \\
\hline$C(3)-C(4)$ & $1.421(5)$ & $\mathrm{C}(18)-\mathrm{P}(1)-\mathrm{C}(1)$ & $103.71(18)$ \\
\hline$C(4)-C(7)$ & $1.410(5)$ & $\mathrm{C}(11)-\mathrm{P}(1)-\mathrm{Au}(1)$ & $107.04(11)$ \\
\hline$C(4)-C(5)$ & $1.427(5)$ & $\mathrm{C}(18)-\mathrm{P}(1)-\mathrm{Au}(1)$ & $109.73(12)$ \\
\hline$C(5)-C(6)$ & $1.423(5)$ & $\mathrm{C}(1)-\mathrm{P}(1)-\mathrm{Au}(1)$ & $122.55(12)$ \\
\hline$C(5)-C(10)$ & $1.424(5)$ & $C(6)-C(1)-C(2)$ & $119.5(3)$ \\
\hline$C(6)-C(6) \# 1$ & $1.500(7)$ & $\mathrm{C}(6)-\mathrm{C}(1)-\mathrm{P}(1)$ & $123.6(3)$ \\
\hline$C(7)-C(8)$ & $1.367(6)$ & $\mathrm{C}(2)-\mathrm{C}(1)-\mathrm{P}(1)$ & $116.6(3)$ \\
\hline$C(8)-C(9)$ & $1.413(5)$ & $C(3)-C(2)-C(1)$ & $121.3(3)$ \\
\hline $\mathrm{C}(9)-\mathrm{C}(10)$ & $1.376(6)$ & $C(2)-C(3)-C(4)$ & $120.7(3)$ \\
\hline$C(11)-C(12)$ & $1.397(5)$ & $C(7)-C(4)-C(3)$ & $122.1(3)$ \\
\hline$C(11)-C(16)$ & $1.400(5)$ & $C(7)-C(4)-C(5)$ & $119.5(3)$ \\
\hline$C(12)-C(13)$ & $1.380(5)$ & $C(3)-C(4)-C(5)$ & $118.4(3)$ \\
\hline$C(13)-C(14)$ & $1.408(5)$ & $C(6)-C(5)-C(10)$ & $122.3(3)$ \\
\hline$C(14)-C(15)$ & $1.382(6)$ & $C(6)-C(5)-C(4)$ & $120.1(3)$ \\
\hline$C(14)-C(17)$ & $1.508(5)$ & $C(10)-C(5)-C(4)$ & $117.6(3)$ \\
\hline$C(15)-C(16)$ & $1.388(5)$ & $C(1)-C(6)-C(5)$ & $119.8(3)$ \\
\hline$C(18)-C(23)$ & $1.384(6)$ & $C(1)-C(6)-C(6) \# 1$ & $122.7(4)$ \\
\hline C(18)-C(19) & $1.393(6)$ & $C(5)-C(6)-C(6) \# 1$ & $117.4(3)$ \\
\hline$C(19)-C(20)$ & $1.372(6)$ & $C(8)-C(7)-C(4)$ & $121.3(3)$ \\
\hline$C(20)-C(21)$ & $1.384(7)$ & $C(7)-C(8)-C(9)$ & $120.0(4)$ \\
\hline $\mathrm{C}(21)-\mathrm{C}(22)$ & $1.386(7)$ & $\mathrm{C}(10)-\mathrm{C}(9)-\mathrm{C}(8)$ & $120.0(4)$ \\
\hline $\mathrm{C}(21)-\mathrm{C}(24)$ & $1.506(6)$ & $C(9)-C(10)-C(5)$ & $121.5(4)$ \\
\hline$C(22)-C(23)$ & $1.385(6)$ & $C(12)-C(11)-C(16)$ & $118.4(3)$ \\
\hline \multicolumn{2}{|c|}{$\mathrm{P}(1)-\mathrm{Au}(1)-\mathrm{Cl}(1)$} & $\mathrm{C}(12)-\mathrm{C}(11)-\mathrm{P}(1)$ & $119.6(3)$ \\
\hline
\end{tabular}




$$
\begin{array}{ll}
\hline \mathrm{C}(16)-\mathrm{C}(11)-\mathrm{P}(1) & 121.7(3) \\
\mathrm{C}(13)-\mathrm{C}(12)-\mathrm{C}(11) & 120.9(3) \\
\mathrm{C}(12)-\mathrm{C}(13)-\mathrm{C}(14) & 120.9(4) \\
\mathrm{C}(15)-\mathrm{C}(14)-\mathrm{C}(13) & 117.8(3) \\
\mathrm{C}(15)-\mathrm{C}(14)-\mathrm{C}(17) & 121.3(3) \\
\mathrm{C}(13)-\mathrm{C}(14)-\mathrm{C}(17) & 120.9(4) \\
\mathrm{C}(14)-\mathrm{C}(15)-\mathrm{C}(16) & 121.7(3) \\
\mathrm{C}(15)-\mathrm{C}(16)-\mathrm{C}(11) & 120.2(3) \\
\mathrm{C}(23)-\mathrm{C}(18)-\mathrm{C}(19) & 118.6(4) \\
\mathrm{C}(23)-\mathrm{C}(18)-\mathrm{P}(1) & 123.5(3) \\
\mathrm{C}(19)-\mathrm{C}(18)-\mathrm{P}(1) & 117.8(3) \\
\mathrm{C}(20)-\mathrm{C}(19)-\mathrm{C}(18) & 120.4(4) \\
\mathrm{C}(19)-\mathrm{C}(20)-\mathrm{C}(21) & 121.3(4) \\
\mathrm{C}(20)-\mathrm{C}(21)-\mathrm{C}(22) & 118.3(4) \\
\mathrm{C}(20)-\mathrm{C}(21)-\mathrm{C}(24) & 121.8(5) \\
\mathrm{C}(22)-\mathrm{C}(21)-\mathrm{C}(24) & 119.8(5) \\
\mathrm{C}(23)-\mathrm{C}(22)-\mathrm{C}(21) & 120.7(4) \\
\mathrm{C}(18)-\mathrm{C}(23)-\mathrm{C}(22) & 120.6(4)
\end{array}
$$


Table 13. Anisotropic displacement parameters $\left(\AA^{2} \times 10^{3}\right)$ for 47. The anisotropic displacement factor exponent takes the form: $-2 \pi^{2}\left[\left(h^{*}\right)^{2} U_{11}+\ldots+2 h k a+b+U_{12}\right]$

$$
\mathrm{U}^{11} \quad \mathrm{U}^{22} \quad \mathrm{U}^{33} \quad \mathrm{U}^{23} \quad \mathrm{U}^{13} \quad \mathrm{U}^{12}
$$

$\begin{array}{lllllll}\mathrm{Au}(1) & 13(1) & 12(1) & 11(1) & -1(1) & -1(1) & 3(1) \\ \mathrm{Cl}(1) & 23(1) & 30(1) & 14(1) & -5(1) & -5(1) & 3(1) \\ \mathrm{P}(1) & 11(1) & 11(1) & 11(1) & 0(1) & -1(1) & 5(1) \\ \mathrm{C}(1) & 9(2) & 13(2) & 18(2) & -1(1) & 0(1) & 6(2) \\ \mathrm{C}(2) & 14(2) & 18(2) & 11(2) & 2(1) & 1(1) & 8(2) \\ \mathrm{C}(3) & 18(2) & 20(2) & 10(2) & -2(1) & -3(1) & 12(2) \\ \mathrm{C}(4) & 14(2) & 13(2) & 18(2) & -4(1) & -2(1) & 10(2) \\ \mathrm{C}(5) & 12(2) & 11(2) & 14(2) & -4(1) & -1(1) & 6(1) \\ \mathrm{C}(6) & 13(2) & 12(2) & 11(2) & 1(1) & 1(1) & 10(2) \\ \mathrm{C}(7) & 16(2) & 19(2) & 22(2) & -7(2) & -6(2) & 9(2) \\ \mathrm{C}(8) & 13(2) & 14(2) & 29(2) & -11(2) & -4(2) & 4(2) \\ \mathrm{C}(9) & 19(2) & 17(2) & 21(2) & 2(2) & 6(2) & 6(2) \\ \mathrm{C}(10) & 18(2) & 15(2) & 19(2) & -1(1) & 4(2) & 10(2) \\ \mathrm{C}(11) & 12(2) & 9(2) & 11(2) & -4(1) & -1(1) & 3(1) \\ \mathrm{C}(12) & 17(2) & 14(2) & 22(2) & 6(1) & 3(2) & 7(2) \\ \mathrm{C}(13) & 17(2) & 18(2) & 27(2) & 4(2) & 4(2) & 9(2) \\ \mathrm{C}(14) & 18(2) & 17(2) & 16(2) & 0(1) & 5(2) & 7(2) \\ \mathrm{C}(15) & 16(2) & 17(2) & 12(2) & 3(2) & 2(1) & 4(2) \\ \mathrm{C}(16) & 17(2) & 19(2) & 13(2) & -2(1) & -5(2) & 10(2) \\ \mathrm{C}(17) & 25(2) & 28(2) & 30(2) & 4(2) & 9(2) & 11(2) \\ \mathrm{C}(18) & 20(2) & 17(2) & 13(2) & 5(1) & 5(1) & 12(2)\end{array}$


C(19) $34(3) \quad 21(2) \quad 18(2) \quad 4(2) \quad 5(2) \quad 17(2)$

C(20) $50(3) \quad 25(2) \quad 23(2) \quad 3(2) \quad 9(2) \quad 26(2)$

C(21) 41(3) $39(3) \quad 24(2) \quad 14(2) \quad 16(2) \quad 32(2)$

C(22) $26(2) \quad 42(3) \quad 30(2) \quad 13(2) \quad 7(2) \quad 25(2)$

C(23) 20(2) 24(2) 26(2) $2(2) \quad 3(2) \quad 13(2)$

C(24) 71(4) 61(4) 46(3) $17(3) \quad 22(3) \quad 58(3)$

Table 14. Hydrogen coordinates $\left(\mathrm{x}_{10} 0^{4}\right)$ and isotropic displacement parameters $\left(\AA^{2} \mathrm{x}\right.$ $10^{3}$ ) for 47.

\begin{tabular}{|c|c|c|c|c|}
\hline & $\mathrm{x}$ & $\mathrm{y}$ & $\mathrm{z}$ & $\mathrm{U}(\mathrm{eq})$ \\
\hline $\mathrm{H}(2)$ & 9378 & 11066 & 4682 & 17 \\
\hline $\mathrm{H}(3)$ & 11315 & 13011 & 4885 & 18 \\
\hline $\mathrm{H}(7)$ & 13370 & 15164 & 4635 & 23 \\
\hline $\mathrm{H}(8)$ & 14634 & 16690 & 4047 & 25 \\
\hline $\mathrm{H}(9)$ & 13837 & 16353 & 3251 & 24 \\
\hline $\mathrm{H}(10)$ & 11851 & 14427 & 3049 & 20 \\
\hline $\mathrm{H}(12)$ & 6168 & 10398 & 4047 & 22 \\
\hline $\mathrm{H}(13)$ & 4384 & 9689 & 4575 & 25 \\
\hline $\mathrm{H}(15)$ & 5474 & 7227 & 5219 & 20 \\
\hline $\mathrm{H}(16)$ & 7249 & 7907 & 4685 & 18 \\
\hline $\mathrm{H}(17 \mathrm{~A})$ & 2704 & 7052 & 5109 & 43 \\
\hline $\mathrm{H}(17 \mathrm{~B})$ & 3270 & 8496 & 5338 & 43 \\
\hline $\mathrm{H}(17 \mathrm{C})$ & 3644 & 7464 & 5565 & 43 \\
\hline $\mathrm{H}(19)$ & 7410 & 6907 & 3629 & 28 \\
\hline
\end{tabular}




\begin{tabular}{lllll}
\hline $\mathrm{H}(20)$ & 8355 & 5557 & 3725 & 35 \\
$\mathrm{H}(22)$ & 11602 & 8617 & 4355 & 35 \\
$\mathrm{H}(23)$ & 10655 & 9983 & 4264 & 27 \\
$\mathrm{H}(24 \mathrm{~A})$ & 11668 & 6592 & 4030 & 72 \\
$\mathrm{H}(24 \mathrm{~B})$ & 10287 & 5300 & 3897 & 72 \\
$\mathrm{H}(24 \mathrm{C})$ & 10624 & 5756 & 4437 & 72
\end{tabular}

(1) (a) Méndez, M.; Muñoz, M. P.; Echavarren, A. M. J. Am. Chem. Soc. 2000, 122, 11549-11550. (b) Méndez, M.; Muñoz, M. P.; Nevado, C.; Cárdenas, D. J.; Echavarren, A. M. J. Am. Chem. Soc. 2001, 123, 10511-10520.

(2) Brunner, H.; Obermann, U. Chem. Ber. 1989, 122, 499-507.

(3) SMART v. 5.625, Area-Detector Software Package; Bruker AXS 1997-2001. Madison, WI.

(4) Sheldrick, G. M. SADABS version 2.03, a Program for Empirical Absorption Correction; Universität Göttingen, 1997-2001.

(5) SAINT + NT ver. 6.04. SAX Area-Detector Integration Program. Bruker AXS 1997-2001. Madison, WI.

(6) Bruker AXS SHELXTL version 6.10. Structure Determination Package. Bruker AXS 2000. Madison, WI.

(7) Sheldrick, G. M. SHELXS-97, Program for Structure Refinement; Universität Göttingen, 1997-2001. 